\title{
THE EQUATIONS OF THE MULTI-PHASE HUMID ATMOSPHERE EXPRESSED AS A QUASI VARIATIONAL INEQUALITY
}

\author{
YINING $\mathrm{CAO}^{\dagger}$, MAKRAM HAMOUDA $^{\dagger, \star}$, ROGER TEMAM $^{\dagger}$, JOSEPH TRIBBIA $^{\ddagger}$, \\ AND XIAOYAN WANG ${ }^{\dagger}$
}

\begin{abstract}
In this article we propose a new formulation of the equations of the humid atmosphere with a multi-phase saturation generalizing thus the model introduced in [TWu15] and [TWa15]. More precisely, we consider the more realistic situation where the humid atmosphere comprises these components, namely water vapor, liquid water and cloud condensates and furthermore the saturation concentration is not constant. With the additional constraint that the vapor mass ratio $q_{v}$ is less than the saturation concentration $q_{v s}$, which depends itself on the state, we are led, from the mathematical point of view, to introduce and handle a system of equations and inequations involving some quasi-variational inequalities for which we prove the existence of solutions.
\end{abstract}

\section{INTRODUCTION}

A phenomenon as common as the clouds is nevertheless far to be understood from the physical point of view and the specialists believe that the clouds (and the aerosols participating in their formation and evolution) is the greatest source of uncertainty regarding the current numerical simulations for weather and climate predictions.

Clouds are made of many components, air, water, liquid water, ice, pollutants, etc.

The mathematical theory of the equations of the humid atmosphere [Gil82], [Ped87] has been initiated in [LTW92] and more recently in [GH06, GH11]. However, in these references, the humidity is only accounted for through the mass fraction $q$ of air vapor; in addition the saturation of water vapor in the air is not accounted for, so that the equation for the concentration $q$ of water vapor in the air is a mere transport equation. To the best of our knowledge the first articles accounting for the water saturation are [CT12], [CFTT13], and [BCT14]. In these articles the existence of a change of phase leads to the introduction of a Heaviside function, so that the equations for $q$ and $T$ (the temperature) appear as nonlinear, discontinuous and non-monotone. Nevertheless results of existence, uniqueness, maximum principle and regularity of solutions were established. For other equations involving a discontinuous Heaviside function in geophysics see e.g. [Dia93, DT99], and [Fei91, FN91, Gil82] in more general contexts.

Date: November 29, 2017.

2010 Mathematics Subject Classification. 35K87, 35Q35, 76D03, 86A10.

Key words and phrases. Humid atmosphere, Penalization, Regularization, Variational and Quasi Variational inequalities. 
Two simplifying assumptions were made in the references [BCT14, CFTT13, CT12]: namely that the velocity of the fluid $\mathbf{u}$ is known and that the saturation concentration $q_{v s}$ is constant. In [CHKTZ], the authors removed the hypothesis that the velocity is known and they studied the coupled system for $q, T, \mathbf{u}$, thus combining the methods in [CFTT13, CT12] with the methods for the 3-dimensional primitive equations (PEs), [CT07], [Kob06].

In the article [TWu15] the authors assumed again that the velocity $\mathbf{u}$ is prescribed but they also assumed, for simplicity, that the saturation concentration $q_{v s}$ is constant. They then observed that the basic equations for $q$ and $T$ as reported e.g. in the classical references [Hal71, HW80, RY89] are inconsistent for the limit values $q=0$ and $q=1$. This difficulty was reported in the geophysics literature in [TT16], and, in [TWu15] and [TT16] the authors propose to resolve the contradiction in the equations by introducing a unilateral equation (an inequality) valid for $q=0$ and $q=1$, and then the whole problem is set as a variational inequality. For general results on variational inequalities and their utilization in mechanics and physics, see e.g. [Bre72, DL76, Fre02, KS80, ET76].

In the present article, we generalize the work of [TWu15] (see also [TWa15]) by considering a more detailed description of the humid atmosphere, namely we assume that the humid quantities comprise the water vapor, the cloud-condensates, and rain water with respective mass densities $q_{v}, q_{c}$ and $q_{r}$. In the earlier works ([TWu15], [TWa15] and before) $q_{v}$ is the quantity which was called $q$ (and $q_{v s}$ was called $q_{v s}$ ). Because of the increased complexity of the model we first recall in Section 2 all the equations, mostly based on the references [Gra98, Hal71, KW78, KBH98, RY89, Xue89] in view also of setting the notations, and putting the equations in a form suitable for mathematical treatment. The mathematical treatment of the problem is conducted in Sections 3 and 4 .

The multi-species model that we consider in this article is described below. Meanwhile, a different model for multi-species humid atmosphere was introduced in [KM06] and studied from the mathematical viewpoint in the recent article [HKLT17]. As explained in [KM06] (see after (9) in [KM06]), it is often assumed in cloud microphysics parameterizations that the vapor-to-cloud water conversion is instantaneous, i.e. that either the air is saturated, such that the water vapor content matches its saturation value, $q_{v}=q_{v s}(T, p)$, and the cloud water droplets can exist with $q_{c}>0$, or the air is undersaturated, i.e. $q_{v}<q_{v s}$, in which case $q_{c} \equiv 0$. See [Gra98] and other references below; this is our point of view here. In [KM06] and [HKLT17], the authors do not assume this limiting behavior from the outset and demonstrate how it may be derived in a consistent asymptotic framework given large but finite condensation rates. This is the main deviation of the bulk microphysics description in [KM06] from the scheme related to [Gra98] that we study here.

\section{Mathematical Formulation of the PROBlem}

Following the references quoted above above, we consider the conservation equations for the relative mass densities $q_{v}, q_{c}, q_{r}$ and for the temperature $\mathrm{T}$ (or more precisely the 
difference $\theta^{\prime}$ between the potential temperature $\theta$ and a reference temperature $\theta_{h}, \theta^{\prime}=$ $\left.\theta-\theta_{h}\right)$.

We let $\mathcal{M} \subset \mathbb{R}^{3}$ be the spatial domain for our study and a typical point in $\mathcal{M}$ is denoted by $\mathbf{x}=(x, y, p)$ where $p$ is the pressure. We use $\rho, q, \theta, T$ and $e_{v s}$ to denote density, concentration, potential temperature, temperature, and saturation vapor pressure, respectively. In earlier works [BCT14], [TWu15], [TWa15], we considered the atmosphere to be a mixture of dry air and water vapor. In our current investigation, we will consider the water vapor, cloud-condensate and rain water for the humid atmosphere so as to include the clouds. For a specific quantity, we shall use the subindices $v, c$, and $r$ to represent this quantity for the water vapor, cloud-condensate and rain water. For example, $\rho_{v}, q_{v}$ represent the density and concentration of water vapor, $\rho_{c}, q_{c}$ the density and concentration of cloud-condensate, and $\rho_{r}, q_{r}$ the density and concentration of rain water, etc.

Assuming the velocity $\mathbf{u}=(u, v, \omega)$ is known, the unknowns for our current study are the potential temperature $\theta$, the concentrations of the water vapor, cloud-condensate and rain water $q_{v}, q_{c}, q_{r}$ and the saturation concentration $q_{v s}$. If $T$ is the temperature then we classically have

$$
\theta=T\left(\frac{p_{0}}{p}\right)^{\kappa}=\frac{T}{\Pi}, \Pi=\left(\frac{p}{p_{0}}\right)^{\kappa},
$$

where $\kappa=(\gamma-1) / \gamma$ and $\gamma=c_{p} / c_{v}$ is the ratio of specific heats at constant pressure and at constant volume.

Before going any further, we shall first make some simple observations. Of course, the quantities $q_{v}, q_{c}, q_{r}, q_{v s}$ being relative mass fractions ratios take their values in the interval $[0,1]$. Furthermore, the air can not be supersaturated (in general). In other words, we have the constraint $0 \leqslant q_{v} \leqslant q_{v s}$.

Following e.g. [MP74] or [KW78] (see in particular (2.5) in [KW78]), the general form of the equations for $q_{v}, q_{c}, q_{r}$ is given by

$$
\frac{d q}{d t}=M_{q}+D_{q}
$$

In (2.2), the symbol $\frac{d}{d t}$ is the material derivative given here by $\partial_{t}+\mathbf{u} \cdot \nabla_{\mathbf{x}}$, i.e.,

$$
\frac{d}{d t}=\frac{\partial}{\partial t}+u \frac{\partial}{\partial x}+v \frac{\partial}{\partial y}+\omega \frac{\partial}{\partial p}
$$

where $\omega=\frac{d p}{d t}$. Corresponding to $q_{v}, q_{c}$ and $q_{r}$, the terms $D_{q}$ are the usual dissipation terms (like the $3 D$ Laplacian $\Delta_{3}$ ) and the quantities $M_{q}$ are the rates of the production of species $q$, which are described below using the notations in [KW78] (see $(2.9 \mathrm{~b})-(2.9 \mathrm{~d}))$ :

$$
\left\{\begin{array}{l}
M_{q_{v}}=\delta \frac{d q_{v s}}{d t}+E_{r}, \\
M_{q_{c}}=-\delta \frac{d q_{v s}}{d t}-A_{r}-C_{r}, \\
M_{q_{r}}=-g \frac{\partial}{\partial p}\left(\rho q_{r} V_{t}\right)-E_{r}+A_{r}+C_{r} .
\end{array}\right.
$$


Note that in (2.4), $\rho$ is given by the ideal gas law:

$$
p=\rho R T, \quad \rho=\frac{p}{R T},
$$

and that $T$ in (2.5) is the total temperature and not the deviation $T^{\prime}$ appearing in (2.12) below; $V_{t}$ appearing in the expression of $M_{q_{r}}$ is the terminal velocity of the falling rain. The term $A_{r}$ is the rate of auto-conversion of the rain water; $C_{r}$ is the rate of the collection of cloud water by falling rain; and $E_{r}$ represents the evaporation rate of the rain water. Most important for our study is the term $\delta \frac{d q_{v s}}{d t}$, which represents the rate of condensation or evaporation of the cloud water, and this occurs only when $\omega<0$ falling water and $q_{v} \geqslant q_{v s}$. Hence the coefficient $\delta$ is defined as:

$$
\begin{aligned}
& \delta=1, \text { if } \omega<0 \text { and } q_{v} \geqslant q_{v s}, \\
& \delta=0, \text { if } \omega \geqslant 0 \text { or } q_{v}<q_{v s} .
\end{aligned}
$$

The function $q_{v s}$ is a diagnostic variable; it is explicitly given at each instant of time as a function of $p$ and $T$ (or $\theta$ ), that is

$$
q_{v s}=Q_{v s}(p, T) .
$$

The expression of $q_{v s}$ as a function of $T$ and $p$ results from the application of the Clausius-Clapeyron equation. According to ([RY89], p. 14), qvs can be expressed as a function of the saturation vapor pressure $e_{v s}$

$$
q_{v s}=\frac{3.8}{p-0.378 e_{v s}} \frac{e_{v s}}{6.11}=0.6219 \frac{e_{v s}}{p-0.378 e_{v s}},
$$

where by Tetens' formula (see (2.11) of [KW78]),

$$
e_{v s}=6.11 \exp \left(a \frac{T-273}{T-b}\right) .
$$

Here $T$ is in Kelvin, $a=17.27, b=35.5$. Because we only consider the above freezing case in our model, $b \ll 273, b \ll T$, and from now on we set $b=0$ for simplicity.

Remark 2.1. We see that $e_{s}$ is a strictly positive, bounded and smooth function of the temperature $T$ for the temperature ranges found in the troposphere. Considering the usual range for pressure $p$, e.g. $200 \leqslant p \leqslant 1000$ (see [BCHTT15]), we can avoid the possible singularity at $p=0.378 e_{v s}$ in (2.8) by a suitable modification of (2.9) outside the physical relevant values of $T\left(\operatorname{see} \varphi(T)\right.$ in Remark 2.3). So $q_{v s}$ is a positive smooth and increasing function of $e_{v s}$, which in turn implies that $q_{v s}=Q_{v s}(p, T)$ is a positive bounded smooth function of $p$ and $T$ for all values of $p \geqslant 0$ and $T \in \mathbb{R}$. In particular, we will use the properties that $Q_{v s}(p, T)$ has bounded first order and second order partial derivatives with respect to the variables $p$ and $T$ in Section 4 .

Now we need to find the expression $d q_{v s} / d t$ which appears in the right-hand sides of equations (2.4) and (2.11). As in [Hal71] and [HW80], the expression of $\frac{d q_{v s}}{d t}$ results from combining the first law of thermodynamics with the Clausius-Clapeyron equations, so that

$$
\frac{d q_{v s}}{d t}=F(p, T)=\frac{q_{v s} T}{p}\left(\frac{L R-c_{p} R_{v} T}{c_{p} R_{v} T^{2}+q_{v s} L^{2}}\right) \omega
$$


where $\mathrm{p}$ and $\mathrm{T}$ are the pressure and temperature; $L$ is the latent heat of vaporization; $R, R_{v}$ are the gas constants for dry air and water vapor respectively and $c_{p}$ represents the specific heat of dry air at constant pressure.

Finally we have to supplement the equations above with the equation for the temperature $T$. In fact, instead of $T$, we consider the potential temperature $\theta$ previously introduced, or more precisely the deviation $\theta^{\prime}=\theta-\theta_{h}$, where $\theta_{h}$ is a reference temperature.

Hence, as with (2.2) and (2.4), the equation for $\theta^{\prime}$ is given by

$$
\frac{d \theta^{\prime}}{d t}=-\frac{\Pi R}{g^{2} \Phi} \omega N_{h}^{2} \theta_{h}+M_{\theta}+D_{\theta}+\frac{\dot{Q}}{c_{p} \Pi},
$$

with

$$
M_{\theta}=-\frac{L}{c_{p} \Pi}\left(\delta \frac{d q_{v s}}{d t}+E_{r}\right)
$$

(see e.g. (2.9a) in [KW78]). Also, following [KW78] (see (2.2) - (2.3) in [KW78] ) we have

$$
\theta^{\prime}=\frac{T^{\prime}}{\Pi}, \quad \theta_{h}=\frac{T_{h}}{\Pi}
$$

where $T=T_{h}+T^{\prime}$; see also equation (6) in [MP74]. Furthermore we have (see [Xue89], $(1.2 .27)-(1.2 .28))$ :

$$
N_{h}^{2}=-\frac{g r_{h}}{\theta_{h}} \frac{\partial \theta_{h}}{\partial p}, r_{h}=g \rho_{h}=\frac{g p}{R T_{h}}
$$

The source terms. The coefficients describing the microphysics $A_{r}, C_{r}, E_{r}$ and the terminal velocity $V_{t}$ are defined empirically. Common expressions of these terms are as follows (see e.g. [KW78]):

$$
\begin{gathered}
A_{r}=k_{1}\left(q_{c}-q_{c r i t}\right)^{+}, C_{r}=k_{2} q_{c} q_{r}^{0.875}, E_{r}=k_{3}\left(q_{r}^{+}\right)^{0.5}\left(q_{v s}-q_{v}\right)^{+}, \\
V_{t}=5.32 q_{r}^{0.2} .
\end{gathered}
$$

We observe that all these quantities are continuous functions of $U=\left(q_{v}, q_{c}, q_{r}, \theta^{\prime}\right)$. We will slightly modify some terms in a way which simplifies the mathematical study but does not modify the physical relevance of the equations. For example, after a suitable extension outside the physical relevant values of $q_{v}, q_{c}, q_{r}, \theta^{\prime}$, all what we need is to assume that the coefficients are continuous bounded functions of $U$, compactly supported in the region of $\mathbb{R}^{3}$ corresponding to $q_{v}, q_{c}, q_{r}$.

Remark 2.2. For mathematical convenience and in agreement with the physical meaning of $q_{r}\left(0 \leqslant q_{r} \leqslant 1\right)$, we will replace $q_{r}$ in $(2.14)$ by $\tau\left(q_{r}\right)=0$ if $q_{r} \leqslant 0 ;=q_{r}$ if $0 \leqslant q_{r} \leqslant 1 ;$ and $=1$ if $q_{r} \geqslant 1$.

Remark 2.3. We similarly need to comment on (2.10) and change its expression outside the physically relevant values of $q_{v s}, T$ and $\omega$. Firstly, since (2.10) is only relevant for 
$\omega<0$, we replace $\omega$ by $-\omega^{-}$. Then to avoid a possible singularity at $T=0$, we replace $T$ by $\varphi(T)$, where $\varphi$ is a smooth (e.g. $C^{2}$ ) positive real function with $\varphi(T)$ :

$$
\begin{cases}=T & \text { for } T_{*} \leqslant T \leqslant T_{* *}, \\ \geqslant T_{*} / 2 & \text { for } T \leqslant T_{*}, \\ =0 & \text { for } T \geqslant 2 T_{* *} .\end{cases}
$$

Here $T_{*}>0$ is smaller than any temperature on earth (e.g. $100 \mathrm{~K}$ ) and $T_{* *}$ is larger than any temperature on earth (e.g. $355 \mathrm{~K})$. We see that the function $F(p, T)$ in $(2.10)$ is actually uniformly bounded in $p$ and $T$. Moreover, replacing $T$ by $\varphi(T)$ in $(2.10)$ and for a given initial value $q_{v s}(0) \geqslant 0$ we see that the modified equation (2.10) gives $q_{v s}$ as a positive smooth $\left(C^{2}\right)$ bounded function of $T$ and $p$ after we integrate (2.10):

$$
q_{v s}(p, T)=Q_{v s}(p, T) \geqslant 0 .
$$

Remark 2.4. Some authors consider, instead of the expression of $E_{r}$ in (2.14),

$$
E_{r}=k_{3} T\left(q_{r}^{+}\right)^{\beta}\left(q_{v s}-q_{v}\right)^{+}, \beta \in(0,1],
$$

see e.g. [HKLT17]. We could likewise consider this form of $E_{r}$ if we replace $T$ by $\varphi(T)$ which is physically equivalent as we already discussed.

The rest of the article is organized as follows. In Sections 3 and 4 we develop the mathematical setting for these equations. Sections 3 is devoted to presenting the general mathematical setting, the initial and boundary conditions, and the handling of the quantity $\delta$ by using Heaviside functions, in continuation of [CT12] and [CFTT13]. In Section 4 , we account for the constraint $q_{v} \leqslant q_{v s}$ and introduce the quasi-variational inequality that we intend to study, that is, prove the existence of its solution. To this aim, we introduce, in Section 4, a penalization procedure, by which we approximate the quasi variational inequality by a relatively standard nonlinear problem which can be treated by classical methods. Note that the use of the penalization method is a convenient mathematical tool and we do not try to give a physical meaning to the penalized problem. Penalization has been introduced by R. Courant [Cou43] and it is very common in Optimization Theory (see e.g. [Cea78], [Kar11] and [PT80]). Then we prove some a priori estimates for the penalized ( $\varepsilon$-regularized) solution, and finally pass to the limit as $\varepsilon \rightarrow 0$ to end up with the existence of the solution for the initial (non regularized) problem. The passage to the limit relies on using some classical compactness results and convex analysis tools. Other properties concerning the solutions such as uniqueness, maximum principle, etc., will be addressed elsewhere.

\section{Discontinuity And Boundary VAlue PROBlem}

In this part and the next one, we will consider the above equations which will be supplemented with initial and boundary conditions. There are two additional issues:

(1) The coefficient $\delta$ which is discontinuous and that we will replace by a Heaviside function, as in [CT12], [CFTT13].

(2) The inequality constraint on the variable $q_{v}: q_{v} \leqslant q_{v s}$. 
The first issue is addressed in this section, the second issue is addressed in Section 4.

We are going to write the differential equations and the boundary value problems for the quantities $q_{v}, q_{c}, q_{r}, \theta^{\prime}$. We assume for simplicity that the velocity $\mathbf{u}=(\mathbf{v}, \omega)$ is prescribed, otherwise we would have to add the equations governing the evolution of $\mathbf{u}$.

The flow takes place in a domain $\mathcal{M}$ in the $(x, y, p)$-space, $\mathcal{M}=\mathcal{M}^{\prime} \times\left(p_{0}, p_{1}\right)$, where $\mathcal{M}^{\prime} \subset \mathbb{R}^{2}$ is smooth and bounded, and $0<p_{0} \leqslant p \leqslant p_{1}$ is the range of values of $p$ that we consider; here $p_{0}, p_{1}$ are two fixed real numbers. We use "n" to denote the outward normal vector field to the boundary $\partial \mathcal{M}$ of $\mathcal{M}$ which consists of three parts $\Gamma_{u}, \Gamma_{i}, \Gamma_{l}$, namely the upper and lower interface with the ocean and the lateral components of the boundary. They are defined by

$$
\begin{aligned}
& \Gamma_{u}=\left\{(x, y, p) \in \overline{\mathcal{M}} ; p=p_{0}\right\}, \\
& \Gamma_{i}=\left\{(x, y, p) \in \overline{\mathcal{M}} ; p=p_{1}\right\}, \\
& \Gamma_{l}=\left\{(x, y, p) \in \overline{\mathcal{M}} ; p_{0} \leqslant p \leqslant p_{1},(x, y) \in \partial \mathcal{M}^{\prime}\right\} .
\end{aligned}
$$

We set $\nabla=\left(\partial_{x}, \partial_{y}\right)$ and $\Delta=\partial_{x}^{2}+\partial_{y}^{2}$ to be the horizontal gradient and horizontal Laplace operators, respectively and $\nabla_{3}=\left(\nabla, \partial_{p}\right), \Delta_{3}=\Delta+\partial_{p}^{2}$ to be the $3 \mathrm{D}$ gradient and Laplace operators, respectively. In this way, the heat and vapor diffusion operators $\mathcal{A}_{\theta}$ and $\mathcal{A}_{q}$ are described as

$$
\mathcal{A}_{\theta}=-\mu_{\theta} \Delta-\nu_{\theta} \partial_{p}\left(\left(\frac{g p}{R \bar{\theta}}\right)^{2} \partial_{p}\right), \mathcal{A}_{q}=-\mu_{q} \Delta-\nu_{q} \partial_{p}\left(\left(\frac{g p}{R \bar{\theta}}\right)^{2} \partial_{p}\right),
$$

where $\mu_{q}, \nu_{q}\left(q \in\left\{q_{v}, q_{c}, q_{r}\right\}\right), \mu_{\theta}, \nu_{\theta}, g, R, c_{p}$ are all positive constants and $\bar{\theta}=\bar{\theta}(p)$ is the average potential temperature over the isobar with pressure $p$. We assume that $\bar{\theta}$ satisfies:

$$
\bar{\theta}_{*} \leqslant \bar{\theta}(p) \leqslant \bar{\theta}^{*},\left|\partial_{p} \bar{\theta}(p)\right| \leqslant M, \text { for some positive constants } \bar{\theta}_{*}, \bar{\theta}^{*}, M \text { and } p \in\left[p_{0}, p_{1}\right] \text {. }
$$

We set $U=\left(q_{v}, q_{c}, q_{r}, \theta^{\prime}\right)$. We will now describe in details the boundary value problem for each of the quantities under consideration.

3.1. The equation for $q_{v}$. The equation for $q_{v}$ is written

$$
\begin{aligned}
\frac{\partial q_{v}}{\partial t}+\mathcal{A}_{q_{v}} q_{v}+\mathbf{v} \cdot \nabla q_{v}+\omega \frac{\partial q_{v}}{\partial p} & \in f_{q_{v}}\left(q_{v}, q_{c}, q_{r}, \theta^{\prime}\right)+F \mathcal{H}\left(q_{v}-q_{v s}\right) \\
& =f_{q_{v}}(U)+F \mathcal{H}\left(q_{v}-q_{v s}\right)
\end{aligned}
$$

where $\mathcal{H}$ is the multi-valued Heaviside function such that $\mathcal{H}=[0,1]$ at 0 and (see $(2.10)$ as well as Remark 2.3):

$$
\begin{gathered}
F=F(T, p)=-\omega^{-} \frac{q_{v s} \varphi(T)}{p}\left(\frac{L R-c_{p} R_{v} \varphi(T)}{c_{p} R_{v} \varphi(T)^{2}+q_{v s} L^{2}}\right), \\
f_{q_{v}}(U)=f_{q_{v}}\left(q_{v}, q_{c}, q_{r}, \theta^{\prime}\right)=E_{r} .
\end{gathered}
$$


We will use the form $E_{r}=k_{3} \tau\left(q_{r}\right)^{0.5}\left(q_{v s}-q_{v}\right)^{+}$as indicated in (2.14). Note that we have replaced $\left(q_{r}\right)^{+}$by $\tau\left(q_{r}\right)$ according to Remark 2.2 .

We consider the following boundary conditions to be associated with the above equation:

$$
\partial_{p} q_{v}=\beta_{v}\left(q_{v *}-q_{v}\right) \text { on } \Gamma_{i}, \quad \partial_{p} q_{v}=0 \text { on } \Gamma_{u}, \quad \partial_{n_{v}} q_{v}=0 \text { on } \Gamma_{l},
$$

where $\partial_{n_{v}}=\partial_{n_{\mathcal{A}_{q}}}$ is the co-normal derivative associated with $\mathcal{A}_{q_{v}}$ which reduces on $\Gamma_{l}$ to

$$
-\mu_{q_{v}} \mathbf{n}_{H} \cdot \nabla q_{v}
$$

where $\mathbf{n}_{H}$ is the horizontal component of the unit outward normal $\mathbf{n}$ on $\mathcal{M}$ (that is the unit outward normal on $\Gamma_{l}$ ).

We also associate with (3.4) the following initial condition

$$
q_{v}(x, y, p, 0)=q_{v 0}(x, y, p) .
$$

In (3.7), $q_{v *}=q_{v *}(x, y, t)$ is a specific humidity distribution at the bottom of the atmosphere and $\beta_{v}$ is a given positive constant.

3.2. The equation for $q_{c}$. The equation for $q_{c}$ is written

$$
\begin{aligned}
\frac{\partial q_{c}}{\partial t}+\mathcal{A}_{q_{c}} q_{c}+\mathbf{v} \cdot \nabla q_{c}+\omega \frac{\partial q_{c}}{\partial p} & \in f_{q_{c}}\left(q_{v}, q_{c}, q_{r}, \theta^{\prime}\right)-F \mathcal{H}\left(q_{v}-q_{v s}\right) \\
& =f_{q_{c}}(U)-F \mathcal{H}\left(q_{v}-q_{v s}\right),
\end{aligned}
$$

where $F, \mathcal{H}$ are defined below (3.4) and

$$
f_{q_{c}}(U)=f_{q_{c}}\left(q_{v}, q_{c}, q_{r}, \theta^{\prime}\right)=-k_{1}\left(q_{c}-q_{c r i t}\right)^{+}-k_{2} q_{c} \tau\left(q_{r}\right)^{0.875} .
$$

Similar to what we did for $E_{r}$, here we have replaced $q_{r}$ in $C_{r}$ by $\tau\left(q_{r}\right)$ (compare to $(2.14))$.

We supplement the above equation with the following natural boundary conditions

$$
\partial_{p} q_{c}=\beta_{c}\left(q_{c *}-q_{c}\right) \text { on } \Gamma_{i}, \quad \partial_{p} q_{c}=0 \text { on } \Gamma_{u}, \quad \partial_{n_{c}} q_{c}=0 \text { on } \Gamma_{l},
$$

and the initial condition

$$
q_{c}(x, y, p, 0)=q_{c 0}(x, y, p) .
$$

In (3.12), $q_{c *}=q_{c *}(x, y, t)$ is a critical specific humidity distribution at the bottom of the atmosphere and $\beta_{c}$ is a given positive constant, and $\partial_{n_{c}} q_{c}$ is defined as $\partial_{n_{v}} q_{v}$ in (3.8).

3.3. The equation for $q_{r}$. The equation for $q_{r}$ is written

$$
\frac{\partial q_{r}}{\partial t}+\mathcal{A}_{q_{r}} q_{r}+\mathbf{v} \cdot \nabla q_{r}+\omega \frac{\partial q_{r}}{\partial p}=-g \frac{\partial}{\partial p}\left(\rho q_{r} V_{t}\right)-E_{r}+A_{r}+C_{r} .
$$

Here, we will continue to use the expression $E_{r}=k_{3} \tau\left(q_{r}\right)^{0.5}\left(q_{v s}-q_{v}\right)^{+}$in accordance with the $q_{v}$-equation. 
By (2.15), we have

$$
\frac{1}{5.32} \frac{\partial}{\partial p}\left(\rho q_{r} V_{t}\right)=\frac{\partial}{\partial p}\left(\frac{p q_{r}^{1.2}}{R \Pi \theta}\right)=\frac{q_{r}^{1.2}}{R \Pi \theta}+\frac{1.2 p q_{r}^{0.2}}{\Pi \theta R} \frac{\partial q_{r}}{\partial p}-\frac{p q_{r}^{1.2}}{R \Pi \theta^{2}}\left(\theta \frac{\kappa}{p}+\frac{\partial \theta_{h}(p)}{\partial p}+\frac{\partial \theta^{\prime}}{\partial p}\right)
$$

Referring to (3.15) and replacing again $q_{r}$ by $\tau\left(q_{r}\right)$, the equation for $q_{r}$ takes the following form

$$
\begin{aligned}
\frac{\partial q_{r}}{\partial t}+\mathcal{A}_{q_{r}} q_{r}+\mathbf{v} \cdot \nabla q_{r}+\omega \frac{\partial q_{r}}{\partial p}= & -5.32 g \frac{\partial}{\partial p}\left(\rho q_{r}^{1.2}\right)-k_{3} \tau\left(q_{r}\right)^{0.5}\left(q_{v s}-q_{v}\right)^{+} \\
& +k_{1}\left(q_{c}-q_{c r i t}\right)^{+}+k_{2} q_{c} \tau\left(q_{r}\right)^{0.875}
\end{aligned}
$$

By the ideal gas law (2.5), the above equation can be further transformed to

$$
\begin{aligned}
\frac{\partial q_{r}}{\partial t}+\mathcal{A}_{q_{r}} q_{r}+\mathbf{v} \cdot \nabla q_{r}+\omega \frac{\partial q_{r}}{\partial p}= & -5.32 g \frac{\partial}{\partial p}\left(\frac{p q_{r}^{1.2}}{R \Pi \theta}\right)-k_{3} \tau\left(q_{r}\right)^{0.5}\left(q_{v s}-q_{v}\right)^{+} \\
& +k_{1}\left(q_{c}-q_{c r i t}\right)^{+}+k_{2} q_{c} \tau\left(q_{r}\right)^{0.875} .
\end{aligned}
$$

By (3.15), we obtain

$$
\frac{\partial q_{r}}{\partial t}+\mathcal{A}_{q_{r}} q_{r}+\mathbf{v} \cdot \nabla q_{r}+\omega \frac{\partial q_{r}}{\partial p}=f_{q_{r}}\left(q_{v}, q_{c}, q_{r}, \theta^{\prime}\right)=f_{q_{r}}(U)
$$

where

$$
\begin{aligned}
f_{q_{r}}(U)= & f_{q_{r}}\left(q_{v}, q_{c}, q_{r}, \theta^{\prime}\right) \\
= & -5.32 g\left(\frac{\tau\left(q_{r}\right)^{1.2}}{R \Pi \theta_{\alpha}}+\frac{1.2 p \tau\left(q_{r}\right)^{0.2}}{R \Pi \theta_{\alpha}} \frac{\partial q_{r}}{\partial p}-\frac{p \tau\left(q_{r}\right)^{1.2}}{R \Pi \theta_{\alpha}^{2}}\left(\theta_{\alpha} \frac{\kappa}{p}+\frac{\partial \theta_{h}(p)}{\partial p}+\frac{\partial \theta^{\prime}}{\partial p}\right)\right) \\
& -k_{3} \tau\left(q_{r}\right)^{0.5}\left(q_{v s}-q_{v}\right)^{+}+k_{1}\left(q_{c}-q_{c r i t}\right)^{+}+k_{2} q_{c} \tau\left(q_{r}\right)^{0.875} .
\end{aligned}
$$

In (3.19) we have also replaced $\theta$ by $\theta_{\wedge \alpha}=\min (\theta, \alpha)$ where $\alpha>0$ is less than any temperature on earth. This is physically relevant and mathematically useful.

We supplement equation (3.18) with the following boundary conditions and initial conditions:

$$
\begin{gathered}
\partial_{p} q_{r}=\beta_{r}\left(q_{r *}-q_{r}\right) \text { on } \Gamma_{i}, \quad \partial_{p} q_{r}=0 \text { on } \Gamma_{u}, \quad \partial_{n_{r}} q_{r}=0 \text { on } \Gamma_{l}, \\
q_{r}(x, y, p, 0)=q_{r 0}(x, y, p) .
\end{gathered}
$$

Here $q_{r *}=q_{r *}(x, y, t)$ is a specific humidity distribution at the bottom of the atmosphere; $\beta_{r}$ is a given positive constant. Also $\partial_{n_{r}} q_{r}$ is defined as $\partial_{n_{v}} q_{v}$ in (3.8).

3.4. The equation for $\theta\left(\sim \theta^{\prime}\right)$. The deviation $\theta^{\prime}$ from the reference state $\theta_{h}(p)$ satisfies the following equation

$$
\begin{aligned}
\frac{\partial \theta^{\prime}}{\partial t}+\mathcal{A}_{\theta} \theta^{\prime}+\mathbf{v} \cdot \nabla \theta^{\prime}+\omega \frac{\partial \theta^{\prime}}{\partial p} & \in f_{\theta^{\prime}}\left(q_{v}, q_{c}, q_{r}, \theta^{\prime}\right)-\frac{L}{c_{p} \Pi} F \mathcal{H}\left(q_{v}-q_{v s}\right) \\
& =f_{\theta^{\prime}}(U)-\frac{L}{c_{p} \Pi} F \mathcal{H}\left(q_{v}-q_{v s}\right),
\end{aligned}
$$


where

$$
f_{\theta^{\prime}}(U)=f_{\theta^{\prime}}\left(q_{v}, q_{c}, q_{r}, \theta^{\prime}\right)=-\frac{\theta_{h} N_{h}^{2}}{g} \omega-\frac{L}{c_{p} \Pi}\left(k_{3} \tau\left(q_{r}\right)^{0.5}\left(q_{v s}-q_{v}\right)^{+}\right)+f_{\theta} .
$$

Here $f_{\theta}=f_{\theta}^{1}+f_{\theta}^{2}$, with $f_{\theta}^{1}$ a source term and $f_{\theta}^{2}=\omega \frac{\partial \theta_{h}(p)}{\partial p}$ after observing that $\mathcal{A}_{\theta} \theta_{h}(p)+$ $\mathbf{v} \cdot \nabla \theta_{h}(p)$ vanishes.

We consider the boundary conditions

$$
\partial_{p} \theta^{\prime}=\alpha\left(\theta_{*}^{\prime}-\theta^{\prime}\right) \text { on } \Gamma_{i}, \quad \partial_{p} \theta^{\prime}=0 \text { on } \Gamma_{u}, \quad \partial_{n_{\theta^{\prime}}} \theta^{\prime}=0 \text { on } \Gamma_{l},
$$

and initial condition

$$
\theta^{\prime}(x, y, p, 0)=\theta_{0}^{\prime}(x, y, p) .
$$

Here the function $\theta_{*}^{\prime}=\theta_{*}^{\prime}(x, y, t)$ is a typical potential temperature; $\alpha$ is a given positive constant, and $\partial_{n_{\theta^{\prime}}} \theta^{\prime}$ is defined as $\partial_{n_{v}} q_{v}$ in (3.8).

\section{VARIATIONAL AND WEAK FORMULATION OF THE PROBLEM.}

From the mathematical point of view, a new difficulty as compared to [CT12], [CFTT13] is the constraint $q_{v} \leqslant q_{v s}$ which leads us to the concept of quasi variational inequality (instead of a variational inequality). Indeed in the notations below the velocity $\mathbf{u}$ is still given and the set of unknowns $U$ consists of $q_{v}, q_{r}, q_{c}$ and the temperature $T$; in fact we rather consider the potential temperature $\theta$, and replace it by the difference $\theta^{\prime}=\theta-\theta_{h}$ between $\theta$ and a reference temperature $\theta_{h}$. Hence $U=\left(q_{v}, q_{r}, q_{c}, \theta^{\prime}\right)$. Now, as recalled in Remark 2.3, the saturation concentration $q_{v s}$ is itself a function of $T$ and $p$, which we express as $q_{v s}=Q_{v s}(p, U)$ or $q_{v s}=Q_{v s}(p, T)$ for simplicity. Hence the constraint $q_{v} \leqslant q_{v s}$ appears as a quasi variational inequality where the solution $U$ is subject to belonging to a convex set which depends itself on the solution:

$$
U \in \mathcal{K}=\mathcal{K}(U) .
$$

Quasi variational inequalities have been introduced by Bensoussan and Lions, motivated by the study of economical problems [BL76], [BL77]; see also [BF78], [BL84], [BL73a], [BL73b], [BL74] and [BL75]. Subsequently quasi variational inequalities have been used for problems in mechanics, physics and imagery, see e.g. [KN07], [Kan14], [Mil14] and [LLBS14].

We start in Section 4.1 by giving the weak form of the problem and then in Section 4.2 we account for the constraint $U \in \mathcal{K}(U)$ and introduce the quasi-variational inequality.

4.1. Notations. We denote as usual $H=L^{2}(\mathcal{M}), V=H^{1}(\mathcal{M})$ and we set $\mathbb{H}=$ $H \times H \times H \times H$ and $\mathbb{V}=V \times V \times V \times V$. We use $(\cdot, \cdot)_{L^{2}}\left(\right.$ regarded the same as $\left.(\cdot, \cdot)_{H}\right)$ and $|\cdot|_{L^{2}}$ to denote the usual scalar product and induced norm in $H$. In the space $V$, we will use $((\cdot, \cdot))$ to denote the scalar product adapted to the problem under investigation

$$
((\varphi, \phi)):=(\nabla \varphi, \nabla \phi)+\left(\partial_{p} \varphi, \partial_{p} \phi\right)+\int_{\Gamma_{i}} \varphi \phi d \Gamma_{i}
$$


and the induced norm is denoted $\|\cdot\|$. The symbol $\langle\cdot, \cdot\rangle$ will denote the duality pair between a Banach space $E$ and its dual space $E^{*}$. Associated with the Navier-Stokes equations, we also use the following standard notations:

$$
\begin{aligned}
& \mathbf{H}=\{\mathbf{u} \in H \times H \times H \mid \operatorname{div} \mathbf{u}=0 \text { and } \mathbf{u} \cdot n=0 \text { on } \partial \mathcal{M}\}, \\
& \mathbf{V}=\{\mathbf{u} \in V \times V \times V \mid \operatorname{div} \mathbf{u}=\mathbf{0} \text { and } \mathbf{u} \cdot \mathbf{n}=\mathbf{0} \text { on } \partial \mathcal{M}\},
\end{aligned}
$$

which will serve as the natural function spaces for the vector field $\mathbf{u}$. In fact we will assume that

$$
\mathbf{u} \in L^{\infty}\left(0, t_{1} ; H^{1}(\mathcal{M})^{3}\right) \cap L^{\infty}\left(\left(0, t_{1}\right) \times \mathcal{M}\right)
$$

In view of deriving the weak (variational) formulation of the boundary value problem, we multiply e.g. the expression $\mathcal{A}_{q_{v}} q_{v}$ by a test function $q_{v}^{b}$. Assuming smoothness and taking into account the boundary conditions (3.7) for $q_{v}$ we find

$$
\begin{aligned}
\left\langle\mathcal{A}_{q_{v}} q_{v}, q_{v}^{b}\right\rangle= & \left(-\mu_{q_{v}} \Delta-\nu_{q_{v}} \partial_{p}\left(\left(\frac{g p}{R \bar{\theta}}\right)^{2} \partial_{p}\right), q_{v}^{b}\right) \\
:= & \mu_{q_{v}}\left(\nabla q_{v}, \nabla q_{v}^{b}\right)_{H}+\nu_{q_{v}} \int_{\mathcal{M}}\left(\frac{g p}{R \bar{\theta}}\right)^{2} \partial_{p} q_{v} \partial_{p} q_{v}^{b} d \mathcal{M} \\
& +\nu_{q_{v}} \int_{\Gamma_{i}}\left(\frac{g p_{1}}{R \bar{\theta}}\right)^{2} \beta_{q_{v}}\left(q_{v}-q_{v *}\right) q_{v}^{b} d \Gamma_{i} .
\end{aligned}
$$

We do the same for $q_{c}, q_{r}$ and $\theta^{\prime}$ and thus

$$
\begin{aligned}
\left\langle\mathcal{A}_{q_{c}} q_{c}, q_{c}^{b}\right\rangle= & \mu_{q_{c}}\left(\nabla q_{c}, \nabla q_{c}^{b}\right)_{H}+\nu_{q_{c}} \int_{\mathcal{M}}\left(\frac{g p}{R \bar{\theta}}\right)^{2} \partial_{p} q_{c} \partial_{p} q_{c}^{b} d \mathcal{M} \\
& +\nu_{q_{c}} \int_{\Gamma_{i}}\left(\frac{g p_{1}}{R \bar{\theta}}\right)^{2} \beta_{q_{c}}\left(q_{c}-q_{c *}\right) q_{c}^{b} d \Gamma_{i}, \\
\left\langle\mathcal{A}_{q_{r}} q_{r}, q_{r}^{b}\right\rangle= & \mu_{q_{r}}\left(\nabla q_{r}, \nabla q_{r}^{b}\right)_{H}+\nu_{q_{r}} \int_{\mathcal{M}}\left(\frac{g p}{R \bar{\theta}}\right)^{2} \partial_{p} q_{r} \partial_{p} q_{r}^{b} d \mathcal{M} \\
& +\nu_{q_{r}} \int_{\Gamma_{i}}\left(\frac{g p_{1}}{R \bar{\theta}}\right)^{2} \beta_{q_{r}}\left(q_{r}-q_{r *}\right) q_{r}^{b} d \Gamma_{i},
\end{aligned}
$$

and

$$
\begin{aligned}
\left\langle\mathcal{A}_{\theta} \theta^{\prime}, \theta^{\prime b}\right\rangle= & \mu_{\theta}\left(\nabla \theta^{\prime}, \nabla \theta^{\prime b}\right)_{H}+\nu_{\theta} \int_{\mathcal{M}}\left(\frac{g p}{R \bar{\theta}}\right)^{2} \partial_{p} \theta^{\prime} \partial_{p} \theta^{\prime b} d \mathcal{M} \\
& +\nu_{\theta} \int_{\Gamma_{i}}\left(\frac{g p_{1}}{R \bar{\theta}}\right)^{2} \alpha\left(\theta^{\prime}-\theta_{*}^{\prime}\right) \theta^{\prime b} d \Gamma_{i} .
\end{aligned}
$$

Consequently, we define the following bilinear forms

$$
\begin{aligned}
a_{\theta}\left(\theta^{\prime}, \theta^{\prime b}\right) & =\mu_{\theta}\left(\nabla \theta^{\prime}, \nabla \theta^{\prime b}\right)_{H}+\nu_{\theta} \int_{\mathcal{M}}\left(\frac{g p}{R \bar{\theta}}\right)^{2} \partial_{p} \theta^{\prime} \partial_{p} \theta^{\prime b} d \mathcal{M}+\nu_{\theta} \alpha \int_{\Gamma_{i}}\left(\frac{g p_{1}}{R \bar{\theta}}\right)^{2} \theta^{\prime} \theta^{\prime b} d \Gamma_{i} \\
a_{q}\left(q, q^{b}\right) & =\mu_{q}\left(\nabla q, \nabla q^{b}\right)_{H}+\nu_{q} \int_{\mathcal{M}}\left(\frac{g p}{R \bar{\theta}}\right)^{2} \partial_{p} q \partial_{p} q^{b} d \mathcal{M}+\nu_{q} \beta_{q} \int_{\Gamma_{i}}\left(\frac{g p_{1}}{R \bar{\theta}}\right)^{2} q q^{b} d \Gamma_{i}
\end{aligned}
$$


Similarly, we define $b\left(\mathbf{u}, \psi, \psi^{b}\right)$ as follows:

$$
b\left(\mathbf{u}, \psi, \psi^{b}\right)=\int_{\mathcal{M}}\left(\mathbf{v} \cdot \nabla \psi+\omega \partial_{p} \psi\right) \psi^{b} d \mathcal{M}
$$

which we will use with $\left(\psi, \psi^{b}\right)=\left(\theta^{\prime}, \theta^{\prime b}\right),\left(q_{v}, q_{v}^{b}\right),\left(q_{r}, q_{r}^{b}\right),\left(q_{c}, q_{c}^{b}\right)$. We recall here that $\mathbf{u}=(\mathbf{v}, \omega)$ is the three dimensional velocity, $\mathbf{v}$ is the horizontal velocity and $\omega$ is the vertical velocity of the air in the $x, y, p$ system.

Analogously, we define the linear functionals:

$$
\begin{gathered}
l_{\theta}\left(\theta^{\prime b}\right)=\nu_{\theta} \alpha \int_{\Gamma_{i}}\left(\frac{g p_{1}}{R \bar{\theta}}\right)^{2} \theta_{*} \theta^{\prime b} d \Gamma_{i}, \quad l_{q}\left(q^{b}\right)=\nu_{q} \beta_{q} \int_{\Gamma_{i}}\left(\frac{g p_{1}}{R \bar{\theta}}\right)^{2} q_{*} q^{b} d \Gamma_{i}, \\
l\left(U^{b}\right)=l_{q_{c}}\left(q_{c}^{b}\right)+l_{q_{v}}\left(q_{v}^{b}\right)+l_{q_{r}}\left(q_{r}^{b}\right)+l_{\theta}\left(\theta^{\prime b}\right),
\end{gathered}
$$

which correspond to the constant terms in $\mathcal{A}_{\theta}, \mathcal{A}_{q}$ and $\mathcal{A}$ respectively.

We introduce the multilinear forms for $U$ and $U^{b}=\left(q_{c}^{b}, q_{v}^{b}, q_{r}^{b}, \theta^{\prime b}\right)$

$$
\begin{gathered}
a\left(U, U^{b}\right)=a_{q_{c}}\left(q_{c}, q_{c}^{b}\right)+a_{q_{v}}\left(q_{v}, q_{c}^{b}\right)+a_{q_{r}}\left(q_{r}, q_{r}^{b}\right)+a_{\theta}\left(\theta^{\prime}, \theta^{\prime b}\right), \\
b\left(\mathbf{u}, U, U^{b}\right)=\int_{\mathcal{M}}\left(\mathbf{u} \cdot \nabla_{x, y, p} U\right) \cdot U^{b} d \mathcal{M} .
\end{gathered}
$$

It is easy to see that

$$
b\left(\mathbf{u}, U, U^{b}\right)=b\left(\mathbf{u}, q_{c}, q_{c}^{b}\right)+b\left(\mathbf{u}, q_{v}, q_{v}^{b}\right)+b\left(\mathbf{u}, q_{r}, q_{r}^{b}\right)+b\left(\mathbf{u}, \theta^{\prime}, \theta^{\prime b}\right) .
$$

In view of $\nabla \cdot \mathbf{u}=0$, we readily see by performing integration by parts that

$$
b(\mathbf{u}, \psi, \psi)=0, \forall \psi \in V .
$$

Before we move further, we first give the following well-known estimates.

More precisely, we have the following lemma concerning the boundedness of the above functionals.

Lemma 4.1. Assume $U=\left(q_{v}, q_{c}, q_{r}, \theta^{\prime}\right), U^{b}=\left(q_{v}^{b}, q_{c}^{b}, q_{r}^{b}, \theta^{\prime b}\right) \in \mathbb{V}$ and $\mathbf{u} \in \mathbf{V}$. There exist universal positive constants $\lambda$ and $\kappa$ such that (q denotes here $q_{v}, q_{c}$ or $q_{r}$ ):

$$
\begin{gathered}
\left|a_{\theta}\left(\theta, \theta^{b}\right)\right| \leqslant \kappa\left\|\theta^{\prime}\right\|\left\|\theta^{b}\right\|, \quad a_{\theta}(\theta, \theta) \geqslant \lambda\|\theta\|^{2} ; \\
\left|a_{q}\left(q, q^{b}\right)\right| \leqslant \kappa\|q\|\left\|q^{b}\right\|, \quad a_{q}(q, q) \geqslant \lambda\|q\|^{2} ; \\
\left|b\left(\mathbf{u}, U, U^{b}\right)\right| \leqslant \kappa\|\mathbf{u}\|_{\mathbf{V}}|U|_{L^{2}}^{\frac{1}{2}}\|U\| \frac{1}{2}\left\|U^{\prime b}\right\| ; \\
\left|l_{\theta}\left(\theta^{\prime b}\right)\right| \leqslant \kappa\left\|\theta^{\prime b}\right\|, \quad\left|l_{q}\left(q^{b}\right)\right| \leqslant \kappa\left\|q^{b}\right\| .
\end{gathered}
$$

The proof of Lemma 4.1 is based on a routine use of the Cauchy-Schwarz inequality and the trace theorem. We shall omit the details here.

It is well-known that the linear operators $A_{\theta}, A_{q}: V \rightarrow V^{*}$ defined through the relations

$$
\left\langle A_{\theta} u, v\right\rangle:=a_{\theta}(u, v),\left\langle A_{q} u, v\right\rangle:=a_{q}(u, v), \forall u, v \in V,
$$

are both bounded linear operators. 
Similarly, the operator $B(\mathbf{u}, U)=(b(\mathbf{u}, U), b(\mathbf{u}, q)): V \times \mathbb{V} \rightarrow \mathbb{V}^{*}$ defined by

$$
\left\langle B(\mathbf{u}, U), U^{b}\right\rangle:=\left(b\left(\mathbf{u}, \theta^{\prime}, \theta^{\prime b}\right), b\left(\mathbf{u}, q, q^{b}\right)\right) \forall \mathbf{u} \in V, U, U^{b} \in \mathbb{V},
$$

where $\mathbb{V}^{*}$ is the dual space of $\mathbb{V}$.

4.2. Weak formulation of the problem. Our equations can be written in the following compact form

$$
\partial_{t} U+\mathcal{A} U+\mathbf{u} \cdot \nabla_{\mathbf{x}} \cdot U \in f\left(U, \theta^{\prime}\right)+\mathcal{F} \mathcal{H}\left(q_{v}-q_{v s}\right),
$$

where $\mathcal{F}$ is the vector $\left(F,-F, 0,-\frac{L}{c_{p} \Pi} F\right)^{t}$.

Alternatively, (4.21) means that there exists single-valued Heaviside function $h_{q_{v}} \in$ $\mathcal{H}\left(q_{v}-q_{v s}\right)$ taking values in $[0,1]$ such that

$$
\partial_{t} U+\mathcal{A} U+\mathbf{u} \cdot \nabla_{\mathbf{x}} \cdot U=f\left(U, \theta^{\prime}\right)+\mathcal{F} h_{q_{v}} .
$$

If we adopt the following notations for $U_{0}=U_{0}(x, y, p), U_{*}=U_{*}(x, y, p)$ and $U=$ $U(x, y, p)$

$$
U_{0}=\left(q_{v 0}, q_{c 0}, q_{r 0}, \theta_{0}^{\prime}\right)^{t}, U_{*}=\left(q_{v *}, q_{c *}, q_{r *}, \theta_{*}^{\prime}\right)^{t},
$$

and define the coefficient matrix $\mathcal{C}=\operatorname{diag}\left\{\beta_{c}, \beta_{v}, \beta_{r}, \alpha\right\}$, then the initial and boundary conditions associated with the system (4.21) can be written as follows

$$
\begin{gathered}
U(x, y, p, 0)=U_{0}(x, y, p), \\
\partial_{p} U=\mathcal{C}\left(U_{*}-U\right) \text { on } \Gamma_{i}, \quad \partial_{n_{\mathcal{A}}} U=0 \text { on } \Gamma_{u} \cup \Gamma_{l},
\end{gathered}
$$

where $\partial_{n_{\mathcal{A}}} U$ is defined componentwise as in (3.8).

For the weak formulation we will treat differently the equations for $\bar{U}=\left(q_{c}, q_{r}, \theta^{\prime}\right)$ and the equation for $q_{v}$ which is subjected to the constraint $q_{v} \leqslant q_{s}$.

For $\bar{U}$, we consider the equations (3.10), (3.18), (3.22) for $q_{c}, q_{r}, \theta^{\prime}$, respectively, and multiply them by test functions $q_{c}^{b}, q_{r}^{b}, \theta^{\prime b}$. Assuming smoothness as before, we obtain in view of (4.3)-(4.5),

$$
\int_{0}^{t_{1}}\left[\left\langle\partial_{t} \bar{U}, \bar{U}^{b}\right\rangle+\bar{a}\left(\bar{U}, \bar{U}^{b}\right)+\bar{b}\left(\mathbf{u}, \bar{U}, \bar{U}^{b}\right)-\bar{l}\left(\bar{U}^{b}\right)\right] d t=\int_{0}^{t_{1}}\left(f(\bar{U})+\overline{\mathcal{F}} h_{q_{v}}, \bar{U}^{b}\right) d t,
$$

for all $\bar{U}^{b} \in L^{2}\left(0, t_{1} ;\left(H^{1}\right)^{3}\right)$ and

$$
\bar{U}(t=0)=\bar{U}_{0}
$$

Recall again that here $\bar{l}$ represents the constant part of the operator $\overline{\mathcal{A}}$ and $\overline{\mathcal{F}}$ represents the vector $\left(-F, 0,-\frac{L}{c_{p} \Pi} F\right)^{t}$.

With the constraint $q_{v} \leqslant q_{v s}$, and by analogy with what was done in [TT16] when $q_{v s}$ is constant and $0 \leqslant q_{v} \leqslant q_{v s}$, we can weaken (3.4) in the form:

$$
\mathcal{L}\left(q_{v}\right) \leqslant f_{q_{v}}(U)+F h_{q_{v}},
$$

where $\mathcal{L}\left(q_{v}\right)$ is the left hand side of (3.4). We note that the equation (3.4) is agreeable and consistent with (4.27) if $q_{v}=q_{v s}$ and $\omega<0$. 
Take now a test function $q_{v}^{b} \leqslant q_{v s}$. We see that pointwise

$$
\left(\mathcal{L}\left(q_{v}\right)-f_{q_{v}}(U)-F h_{q_{v}}\right)\left(q_{v}^{b}-q_{v s}\right) \geqslant 0,
$$

in all cases, that is if $q_{v}^{b}=q_{v s}$ or $q_{v}^{b}<q_{v s}$.

This leads us to the formulation of (3.4)-(3.6) as a quasi-variational inequality : $q_{v} \in$ $L^{\infty}\left(0, t_{1} ; L^{2}(\mathcal{M})\right) \cap L^{2}\left(0, t_{1} ; H^{1}(\mathcal{M})\right), q_{v} \leqslant q_{v s}=Q_{v s}(p, T)$ and

$$
\begin{array}{r}
\int_{0}^{t_{1}}\left[\left\langle\partial_{t} q_{v}, q_{v}^{b}-q_{v}\right\rangle+a_{q_{v}}\left(q_{v}, q_{v}^{b}-q_{v}\right)+b\left(\mathbf{u}, q_{v}, q_{v}^{b}-q_{v}\right)-l_{q_{v}}\left(q_{v}^{b}-q_{v}\right)\right] d t \\
\geqslant \int_{0}^{t_{1}}\left(f_{q_{v}}(U)+F h_{q_{v}}, q_{v}^{b}-q_{v}\right) d t
\end{array}
$$

for all $q_{v}^{b} \in L^{\infty}\left(0, t_{1} ; H^{1}\right)$ with $q_{v}^{b} \leqslant q_{v s}=Q_{v s}(p, T)$.

In addition,

$$
q_{v}(t=0)=q_{v 0} .
$$

At this point, let us introduce what we will call here a solution of (4.21) in the weak sense. Let $U_{0} \in \mathbb{V}$ be such that $0 \leqslant q_{v 0} \leqslant q_{s 0}$ and let $t_{1}>0$ be an arbitrary but fixed constant. A vector $U=U(t)=\left(q_{v}, \bar{U}\right) \in L^{2}\left(0, t_{1} ; \mathcal{K}\right) \cap C\left(\left[0, t_{1}\right] ; \mathbb{V}\right)$ with $\partial_{t} \bar{U} \in$ $L^{2}\left(0, t_{1} ;\left(V^{3}\right)^{*}\right), \partial_{t} q_{v} \in L^{5 / 3}\left(0, t_{1} ; V^{*}\right)$ is a solution to the initial-boundary value problem (4.21)-(4.23)-(4.24), if, for almost every $t \in\left[0, t_{1}\right]$ and for every $U^{b} \in \mathcal{K}$, we have $(4.25)$ and (4.28) satisfied.

We recall here that $q_{v s}$ is given by (2.8)-(2.10).

4.3. The penalized and regularized problem. To deal with the inequality constraint $q_{v} \leqslant q_{v s}$ and the discontinuity of the Heaviside function $\mathcal{H}$, we introduce a penalized and regularized version of the problem associated with the parameters $\varepsilon_{1}, \varepsilon_{2}>0$. The penalization is introduced below by introduction of the term $\varepsilon_{1}^{-1}\left(\left(q_{v}-q_{v s}\right)^{+}\right)^{3 / 2}$. We address the discontinuity of the Heaviside function as in [CFTT13] and [CT12]. Recall the multi-valued Heaviside function

$$
\mathcal{H}(r)= \begin{cases}0 & \text { for } r<0 \\ {[0,1]} & \text { for } r=0, \\ 1 & \text { for } r>0\end{cases}
$$

and the single-valued function $h_{q_{v}}$ where $h_{q_{v}} \in \mathcal{H}\left(q_{v}-q_{v s}\right)$. Following [TWu15], we can characterize $h_{q_{v}} \in \mathcal{H}\left(q_{v}-q_{v s}\right)$ by

$$
\left(\left[q_{v}^{b}-q_{v s}\right]^{+}, 1\right)-\left(\left[q_{v}-q_{v s}\right]^{+}, 1\right) \geqslant\left\langle h_{q_{v}}, q_{v}^{b}-q_{v}\right\rangle \text { for a.e. } t \in\left[0, t_{1}\right], \forall q_{v}^{b} \in V .
$$


Now we approximate $h_{q_{v}}$ by $\mathcal{H}_{\varepsilon_{2}}\left(q_{v}-q_{v s}\right)$ for $\varepsilon_{2}>0$, where $\mathcal{H}_{\varepsilon_{2}}(r)$ is defined as

$$
\mathcal{H}_{\varepsilon_{2}}(r)= \begin{cases}0 & \text { for } r \leqslant 0, \\ r / \varepsilon_{2} & \text { for } r \in\left(0, \varepsilon_{2}\right], \\ 1 & \text { for } r>\varepsilon_{2} .\end{cases}
$$

In this setting, $F h_{q_{v}}(\sim F \mathcal{H})$ in the right hand side of (3.4) and (4.28) are replaced by $F \mathcal{H}_{\varepsilon_{2}}\left(q_{v}-q_{v s}\right)$. Similarly, the $h_{q_{v}}(\sim \mathcal{H})$ in the equations for $\theta^{\prime}, q_{c}$ and $q_{r}$ are replaced by $\mathcal{H}_{\varepsilon_{2}}\left(q_{v}-q_{v s}\right)$ as well. Here the regularized $f(U)+\mathcal{F H}_{\varepsilon_{2}}\left(q_{v}-q_{v s}\right)$ has the same boundedness as the original one. Now the related penalized and regularized system of equations reads

$$
\left\{\begin{array}{l}
\partial_{t} q_{v}+\mathcal{A}_{v} q_{v}+\mathbf{v} \cdot \nabla q_{v}+\omega \frac{\partial q_{v}}{\partial p}+\frac{1}{\varepsilon_{1}}\left(\left(q_{v}-q_{v s}\right)^{+}\right)^{3 / 2}=f_{q_{v}}(U)+F \mathcal{H}_{\varepsilon_{2}}\left(q_{v}-q_{v s}\right), \\
\partial_{t} q_{c}+\mathcal{A}_{c} q_{c}+\mathbf{v} \cdot \nabla q_{c}+\omega \frac{\partial q_{c}}{\partial p}=f_{q_{c}}(U)-F \mathcal{H}_{\varepsilon_{2}}\left(q_{v}-q_{v s}\right) \\
\partial_{t} q_{r}+\mathcal{A}_{r} q_{r}+\mathbf{v} \cdot \nabla q_{r}+\omega \frac{\partial q_{r}}{\partial p}=f_{q_{r}}(U) \\
\partial_{t} \theta^{\prime}+\mathcal{A}_{\theta} \theta^{\prime}+\mathbf{v} \cdot \nabla \theta^{\prime}+\omega \frac{\partial \theta^{\prime}}{\partial p}=f_{\theta^{\prime}}(U)-\frac{L}{c_{p} \Pi} F \mathcal{H}_{\varepsilon_{2}}\left(q_{v}-q_{v s}\right) .
\end{array}\right.
$$

It is worth noticing that the unknown functions above depend on the small parameter $\varepsilon=\left(\varepsilon_{1}, \varepsilon_{2}\right)$. Therefore, we will use the notation $U^{\varepsilon}=\left(q_{v}^{\varepsilon}, q_{c}^{\varepsilon}, q_{r}^{\varepsilon}, \theta^{\prime} \varepsilon\right)$, etc. The initial and boundary conditions associated with (4.33) are the same for $U$ :

$$
\begin{gathered}
U^{\varepsilon}(x, y, p, 0)=U_{0}(x, y, p), \\
\partial_{p} U^{\varepsilon}=\mathcal{C}\left(U_{*}-U\right) \text { on } \Gamma_{i}, \quad \partial_{n_{\mathcal{A}}} U^{\varepsilon}=0 \text { on } \Gamma_{u} \cup \Gamma_{l} .
\end{gathered}
$$

To reveal the structural properties of the systems (4.21) and (4.33), we first consider the products related to the nonlinearity $f(U)+\mathcal{F} \mathcal{H}_{\varepsilon_{2}}\left(q_{v}-q_{v s}\right)$, i.e., the following quantities

$$
\begin{gathered}
\left(f_{q_{c}}(U)-F \mathcal{H}_{\varepsilon_{2}}\left(q_{v}-q_{v s}\right), q_{c}\right),\left(f_{q_{v}}(U)+F \mathcal{H}_{\varepsilon_{2}}\left(q_{v}-q_{v s}\right), q_{v}\right), \\
\left(f_{q_{r}}(U), q_{r}\right),\left(f_{\theta^{\prime}}(U)-\frac{L}{c_{p} \Pi} F \mathcal{H}_{\varepsilon_{2}}\left(q_{v}-q_{v s}\right), \theta^{\prime}\right),
\end{gathered}
$$

where $U \in \mathbb{V}$. By analogy with (4.25) the weak formulation of this problem is to find a function $U^{\varepsilon}=U^{\varepsilon}(t)=\left(q_{v}^{\varepsilon}, q_{c}^{\varepsilon}, q_{r}^{\varepsilon}, \theta^{\prime} \varepsilon\right) \in L^{2}\left(0, t_{1} ; \mathbb{V}\right)$ with $\partial_{t} \bar{U}^{\varepsilon} \in L^{2}\left(0, t_{1} ;\left(V^{3}\right)^{*}\right)$ and $\partial_{t} q_{v}^{\varepsilon} \in L^{5 / 3}\left(0, t_{1} ; V^{*}\right)$, such that

$$
\begin{array}{r}
\int_{0}^{t_{1}}\left[\left\langle\partial_{t} U^{\varepsilon}, U^{b}\right\rangle+a\left(U^{\varepsilon}, U^{b}\right)+b\left(\mathbf{u}, U^{\varepsilon}, U^{b}\right)-l\left(U^{b}\right)+\frac{1}{\varepsilon_{1}}\left\langle\left(\left(q_{v}^{\varepsilon}-q_{s}^{\varepsilon}\right)^{+}\right)^{3 / 2}, q_{v}^{b}\right\rangle\right] d t \\
=\int_{0}^{t_{1}}\left(f\left(U^{\varepsilon}\right)+\mathcal{F} \mathcal{H}_{\varepsilon_{2}}\left(q_{v}-q_{v s}\right), U^{b}\right) d t,
\end{array}
$$

for all $\bar{U}^{b} \in L^{2}\left(0, t_{1} ; V^{3}\right)$ and $q_{v}^{b} \in L^{\infty}\left(0, t_{1} ; V\right)$.

$$
U^{\varepsilon}(0)=U_{0}
$$


4.4. The formal a priori estimates and existence of solution for (4.36). We set $U^{b}=U^{\varepsilon}$ in (4.36) and we deduce a new energy equality which is in fact obtained by adding the corresponding energy equalities for each component of $U^{\varepsilon}$, namely $q_{v}^{\varepsilon}, q_{c}^{\varepsilon}, q_{r}^{\varepsilon}$ and $\theta^{\prime} \varepsilon$. For example, the energy equality for $q_{c}$ is obtained by multiplying $(4.33)_{2}$ by $q_{c}$ and integrating over $\mathcal{M}$, etc.

From now, aiming to simplify the presentation, we will omit the dependence on $\varepsilon$ of $U^{\varepsilon}$ that we will denote instead by $U$; the superscript $\varepsilon$ will be reintroduced when it is necessary. Hence, for $q_{c}$, using (4.16)-(4.18), we obtain

$$
\frac{1}{2} \frac{d}{d t}\left|q_{c}\right|_{L^{2}}^{2}+\left(\mathcal{A}_{q_{c}} q_{c}, q_{c}\right)+\left(\mathbf{v} \cdot \nabla q_{c}, q_{c}\right)+\left(\omega \frac{\partial q_{c}}{\partial p}, q_{c}\right)=\left(f_{q_{c}}(U)-F \mathcal{H}_{\varepsilon_{2}}\left(q_{v}-q_{v s}\right), q_{c}\right) .
$$

The other energy equations can be treated similarly except for the RHS of the analogous equations similar to (4.38).

Now, we start by computing the terms in the LHS of (4.38). Hence, using the definition of $\mathcal{A}_{q_{c}}$ as in (3.2) and integrating by parts, we deduce that

$$
\begin{aligned}
\left(\mathcal{A}_{q_{c}} q_{c}, q_{c}\right)= & \left(-\mu_{q_{c}} \Delta q_{c}-\nu_{q_{c}} \partial_{p}\left(\left(\frac{g p}{R \bar{\theta}}\right)^{2} \partial_{p}\right) q_{c}, q_{c}\right) \\
= & \mu_{q_{c}}\left|\nabla q_{c}\right|_{L^{2}}^{2}+\mu_{q_{c}} \int_{\Gamma_{l}} \frac{\partial q_{c}}{\partial n} q_{c} d \Gamma_{l}+\nu_{q_{c}}\left|\frac{g p}{R \bar{\theta}} \frac{\partial q_{c}}{\partial p}\right|^{2} \\
& +\nu_{q_{c}} \int_{\Gamma_{u}}\left(\frac{g p}{R \bar{\theta}}\right)^{2} \partial_{p} q_{c} q_{c} d \Gamma_{u}-\nu_{q_{c}} \int_{\Gamma_{i}}\left(\frac{g p}{R \bar{\theta}}\right)^{2} \partial_{p} q_{c} q_{c} d \Gamma_{i} .
\end{aligned}
$$

Then, we simply observe that, thanks to (3.12), the second and fourth terms in the RHS of (4.39) vanish. We use again the boundary conditions (3.12) to replace the value of the last term in the RHS of (4.39), and we infer that

$$
\begin{aligned}
\left(\mathcal{A}_{q_{c}} q_{c}, q_{c}\right)= & \mu_{q_{c}}\left|\nabla q_{c}\right|_{L^{2}}^{2}+\nu_{q_{c}}\left|\frac{g p}{R \bar{\theta}} \frac{\partial q_{c}}{\partial p}\right|_{L^{2}}^{2} \\
& +\nu_{q_{c}} \beta_{c} \int_{\Gamma_{i}}\left(\frac{g p}{R \bar{\theta}}\right)^{2} q_{c}^{2} d \Gamma_{i}-\nu_{q_{c}} \beta_{c} \int_{\Gamma_{i}}\left(\frac{g p}{R \bar{\theta}}\right)^{2} q_{c} q_{c *} d \Gamma_{i} .
\end{aligned}
$$

The second term in the LHS of (4.38) can be computed using again the integration by parts formula. Hence we have

$$
\begin{aligned}
\left(\mathbf{v} \cdot \nabla q_{c}, q_{c}\right) & =-\frac{1}{2} \int_{\mathcal{M}} \operatorname{div} \mathbf{v} q_{c}^{2} d \mathcal{M}-\frac{1}{2} \int_{\Gamma_{l}}\left(q_{c}\right)^{2} \mathbf{v} \cdot \mathbf{n} d \Gamma_{l} \\
& =(\operatorname{since} \mathbf{u} \cdot \mathbf{n}=0 \text { on } \partial \mathcal{M} \text { by definition of } \mathbf{H}) \\
& =-\frac{1}{2} \int_{\mathcal{M}} \operatorname{div} \mathbf{v} q_{c}^{2} d \mathcal{M}
\end{aligned}
$$

We now calculate the last term in the LHS of (4.38) which reads as follows:

$$
\left(\omega \frac{\partial q_{c}}{\partial p}, q_{c}\right)=-\int_{\mathcal{M}} \frac{\partial \omega}{\partial p} q_{c}^{2} d \mathcal{M}-\int_{\mathcal{M}} \omega \frac{\partial q_{c}}{\partial p} q_{c} d \mathcal{M},
$$


and this yields

$$
\left(\omega \frac{\partial q_{c}}{\partial p}, q_{c}\right)=-\frac{1}{2} \int_{\mathcal{M}} \frac{\partial \omega}{\partial p} q_{c}^{2} d \mathcal{M}
$$

We recall here that the velocity $\mathbf{u}=(\mathbf{v}, \omega)$ satisfies $\mathbf{u} \cdot \mathbf{n}=0$ on $\partial \mathcal{M}$ and $\nabla \cdot \mathbf{u}=$ $\operatorname{div} \mathbf{v}+\partial \omega / \partial p=0$ in $\mathcal{M}$. This implies in particular that

$$
\left(\mathbf{v} \cdot \nabla q_{c}, q_{c}\right)+\left(\omega \frac{\partial q_{c}}{\partial p}, q_{c}\right)=0
$$

Now, combining (4.40) and (4.43) in (4.38), we deduce that

$$
\begin{gathered}
\frac{1}{2} \frac{d}{d t}\left|q_{c}\right|_{L^{2}}^{2}+\mu_{q_{c}}\left|\nabla q_{c}\right|_{L^{2}}^{2}+\nu_{q_{c}}\left|\frac{g p}{R \bar{\theta}} \frac{\partial q_{c}}{\partial p}\right|_{L^{2}}^{2}+\nu_{q_{c}} \beta_{c} \int_{\Gamma_{i}}\left(\frac{g p}{R \bar{\theta}}\right)^{2} q_{c}^{2} d \Gamma_{i} \\
=\nu_{q_{c}} \beta_{c} \int_{\Gamma_{i}}\left(\frac{g p}{R \bar{\theta}}\right)^{2} q_{c} q_{c *} d \Gamma_{i}+\left(f_{q_{c}}(U)-F \mathcal{H}_{\varepsilon_{2}}\left(q_{v}-q_{v s}\right), q_{c}\right) .
\end{gathered}
$$

At this level we are able to estimate the RHS of (4.44) starting by its first term. For that purpose, we use the Cauchy-Schwarz inequality and the identity $2 a b \leqslant a^{2}+b^{2}$. We then infer that

$$
\nu_{q_{c}} \beta_{c} \int_{\Gamma_{i}}\left(\frac{g p}{R \bar{\theta}}\right)^{2}\left|q_{c} q_{c *}\right| d \Gamma_{i} \leqslant \frac{\nu_{q_{c}} \beta_{c}}{2} \int_{\Gamma_{i}}\left(\frac{g p}{R \bar{\theta}}\right)^{2} q_{c}^{2} d \Gamma_{i}+\frac{\nu_{q_{c}} \beta_{c}}{2} \int_{\Gamma_{i}}\left(\frac{g p}{R \bar{\theta}}\right)^{2} q_{c *}^{2} d \Gamma_{i} .
$$

Finally, we observe that, on the one hand, a part of the last term in the RHS of (4.44) is negative, and, on the other hand, the remaining part can be handled using some estimates for $F$ and the Cauchy-Schwarz inequality. Indeed, using (3.11), we have

$$
\left(f_{q_{c}}(U), q_{c}\right)=-k_{1}\left(\left(q_{c}-q_{c r i t}\right)^{+}, q_{c}\right)-k_{2}\left(q_{c} \tau\left(q_{r}\right)^{0.875}, q_{c}\right)
$$

where the RHS of (4.46) are negative since $k_{1}, k_{2} \geqslant 0$, and using the definition of $\left(q_{c}-q_{\text {crit }}\right)^{+}$and of $\tau\left(q_{r}\right)$. Furthermore, we use the fact that $\mathcal{H}_{\varepsilon_{2}}\left(q_{v}-q_{v s}\right)$ and $F$ are uniformly bounded (see remark 2.3$)$ to estimate the quantity $\left(-F \mathcal{H}_{\varepsilon_{2}}\left(q_{v}-q_{v s}\right), q_{c}\right)$. We deduce that

$$
\begin{aligned}
\left|\left(-F \mathcal{H}_{\varepsilon_{2}}\left(q_{v}-q_{v s}\right), q_{c}\right)\right| & \leqslant \mathcal{H}_{\varepsilon_{2}}\left(q_{v}-q_{v s}\right)|F|_{\infty}\left|q_{c}\right| \\
& \leqslant \kappa_{1}\left|q_{c}\right|^{2}+\kappa_{2},
\end{aligned}
$$

where $\kappa$ and the $\kappa_{i}$ are generic constants independent of $\varepsilon$ and taking different values at different places.

Therefore, combining (4.45), (4.46) and (4.47) in (4.44) we conclude that

$$
\begin{aligned}
\frac{1}{2} \frac{d}{d t}\left|q_{c}\right|_{L^{2}}^{2}+\mu_{q_{c}}\left|\nabla q_{c}\right|_{L^{2}}^{2}+\nu_{q_{c}}\left|\frac{g p}{R \bar{\theta}} \frac{\partial q_{c}}{\partial p}\right|_{L^{2}}^{2}+ & \frac{\nu_{q_{c}} \beta_{c}}{2} \int_{\Gamma_{i}}\left(\frac{g p}{R \bar{\theta}}\right)^{2} q_{c}^{2} d \Gamma_{i} \\
+\left(k_{1}\left(q_{c}-q_{c r i t}\right)^{+}+k_{2} q_{c}\left|q_{r}\right|^{0.875}, q_{c}\right) & \leqslant \frac{\nu_{q_{c}} \beta_{c}}{2} \int_{\Gamma_{i}}\left(\frac{g p}{R \bar{\theta}}\right)^{2} q_{c *}^{2} d \Gamma_{i}+\kappa_{1}\left|q_{c}\right|^{2}+\kappa_{2} \\
& \leqslant \kappa_{1}\left|q_{c}\right|^{2}+\kappa_{2} .
\end{aligned}
$$


Using the Gronwall inequality, we conclude, for $q_{c}=q_{c}^{\varepsilon}$, that

$$
\begin{aligned}
& \left|q_{c}^{\varepsilon}\right|_{L^{\infty}\left(0, t_{1} ; L^{2}(\mathcal{M})\right)} \leqslant \kappa, \\
& \left|q_{c}^{\varepsilon}\right|_{L^{2}\left(0, t_{1} ; H^{1}(\mathcal{M})\right)} \leqslant \kappa,
\end{aligned}
$$

where $\kappa$ and the $\kappa_{i}$ are constants independent of $\varepsilon$ as mentioned above.

The same conclusions as in (4.49)-(4.50) hold for $q_{v}^{\varepsilon}, q_{r}^{\varepsilon}$ and $\theta^{\prime} \varepsilon$. Although the energy identity for $q_{v}^{\varepsilon}$ contains a penalization term as stated in (4.33), this term does not affect the analysis above because of its positivity, namely we have $\left(\frac{1}{\varepsilon_{1}}\left(\left(q_{v}-q_{v s}\right)^{+}\right)^{3 / 2}, q_{v}\right) \geqslant 0$ since $q_{v s} \geqslant 0$. Nevertheless, the estimates for the terms $q_{v}^{\varepsilon}, q_{r}^{\varepsilon}, \theta^{\prime} \varepsilon$ need a slightly different treatment for the corresponding right hand sides as they depend on the function $f(U)+\mathcal{F H}_{\varepsilon_{2}}\left(q_{v}-q_{v s}\right)$ and the corresponding value is different for each component of $U$.

In the following we will explain how to treat these terms and the emphasis will be on the differences regarding the analysis done above for $q_{c}$. First, let us start by the $q_{v}$-equation $(4.33)_{1}$. After taking the inner product of $(4.33)_{1}$ with $q_{v}$, we end with the same equation as (4.44) which reads, using (3.6), as follows

$$
\begin{aligned}
& \frac{1}{2} \frac{d}{d t}\left|q_{v}\right|_{L^{2}}^{2}+\mu_{q_{v}}\left|\nabla q_{v}\right|_{L^{2}}^{2}+\nu_{q_{v}}\left|\frac{g p}{R \bar{\theta}} \frac{\partial q_{v}}{\partial p}\right|_{L^{2}}^{2}+\nu_{q_{v}} \beta_{v} \int_{\Gamma_{i}}\left(\frac{g p}{R \bar{\theta}}\right)^{2} q_{v}^{2} d \Gamma_{i} \\
& +\frac{1}{\varepsilon_{1}} \int_{\mathcal{M}}\left(\left(q_{v}-q_{v s}\right)^{+}\right)^{3 / 2} q_{v} d \mathcal{M}=\nu_{q_{v}} \beta_{v} \int_{\Gamma_{i}}\left(\frac{g p}{R \bar{\theta}}\right)^{2} q_{v} q_{v *} d \Gamma_{i} \\
& +\left(F \mathcal{H}_{\varepsilon_{2}}\left(q_{v}-q_{v s}\right)+k_{3} \tau\left(q_{r}\right)^{0.5}\left(q_{v s}-q_{v}\right)^{+}, q_{v}\right) .
\end{aligned}
$$

Using the facts that $q_{v s}$ and $\tau\left(q_{r}\right)$ are bounded as stated in Remarks 2.1 and 2.2, we have

$$
\left|\left(k_{3} \tau\left(q_{r}\right)^{0.5}\left(q_{v s}-q_{v}\right)^{+}, q_{v}\right)\right| \leqslant \kappa_{1}\left|q_{v}\right|^{2}+\kappa_{2} .
$$

The other terms in the RHS of (4.51) can be estimated as we did in (4.45) and (4.47), and we conclude that

$$
\begin{gathered}
\frac{1}{2} \frac{d}{d t}\left|q_{v}\right|_{L^{2}}^{2}+\mu_{q_{v}}\left|\nabla q_{v}\right|_{L^{2}}^{2}+\nu_{q_{v}}\left|\frac{g p}{R \bar{\theta}} \frac{\partial q_{v}}{\partial p}\right|_{L^{2}}^{2}+\nu_{q_{v}} \beta_{v} \int_{\Gamma_{i}}\left(\frac{g p}{R \bar{\theta}}\right)^{2} q_{v}^{2} d \Gamma_{i} \\
+\frac{1}{\varepsilon_{1}} \int_{\mathcal{M}}\left(\left(q_{v}-q_{v s}\right)^{+}\right)^{3 / 2} q_{v} d \mathcal{M} \leqslant \kappa_{1}\left|q_{v}\right|^{2}+\kappa_{2},
\end{gathered}
$$

and the Gronwall Lemma implies the desired estimates, namely

$$
\begin{aligned}
& \left|q_{v}^{\varepsilon}\right|_{L^{\infty}\left(0, t_{1} ; L^{2}(\mathcal{M})\right)} \leqslant \kappa, \\
& \left|q_{v}^{\varepsilon}\right|_{L^{2}\left(0, t_{1} ; H^{1}(\mathcal{M})\right)} \leqslant \kappa .
\end{aligned}
$$

We also have

$$
\begin{aligned}
& \frac{1}{\varepsilon_{1}} \int_{0}^{t_{1}} \int_{\mathcal{M}}\left(\left(q_{v}-q_{v s}\right)^{+}\right)^{3 / 2} q_{v} d \mathcal{M} d s=\frac{1}{\varepsilon_{1}} \int_{0}^{t_{1}} \int_{\mathcal{M}}\left[\left(q_{v}-q_{v s}\right)^{+}\right]^{5 / 2} d \mathcal{M} d s+ \\
& \frac{1}{\varepsilon_{1}} \int_{0}^{t_{1}} \int_{\mathcal{M}}\left(\left(q_{v}-q_{v s}\right)^{+}\right)^{3 / 2} q_{v s} d \mathcal{M} d s \leqslant \kappa,
\end{aligned}
$$


and since $q_{v s} \geqslant 0$,

$$
\frac{1}{\varepsilon_{1}} \int_{0}^{t_{1}} \int_{\mathcal{M}}\left[\left(q_{v}-q_{v s}\right)^{+}\right]^{5 / 2} d \mathcal{M} d s \leqslant \kappa
$$

More estimates about the penalization term resulting from (4.53),(4.54) and (4.55) will be deduced below (see Lemma 4.2).

Then, we consider first the equation of $\theta^{\prime}$ and let the equation of $q_{r}$ to the end since the RHS of its equation depends on $\partial \theta^{\prime} / \partial p$ as stated in (3.19). Thus we multiply the equation of $\theta^{\prime}$, given by $(4.33)_{4}$, by $\theta^{\prime}$ and integrate over $\mathcal{M}$. Since the boundary conditions for $\theta^{\prime}$ are the same as those of $q_{c}$, we obtain an equation similar to (4.44). Remembering also (3.23), we arrive at

$$
\begin{aligned}
& \frac{1}{2} \frac{d}{d t}\left|\theta^{\prime}\right|_{L^{2}}^{2}+\mu_{\theta^{\prime}}\left|\nabla \theta^{\prime}\right|_{L^{2}}^{2}+\nu_{\theta^{\prime}}\left|\frac{g p}{R \bar{\theta}} \frac{\partial \theta^{\prime}}{\partial p}\right|_{L^{2}}^{2}+\nu_{\theta^{\prime}} \alpha \int_{\Gamma_{i}}\left(\frac{g p}{R \bar{\theta}}\right)^{2} \theta^{\prime 2} d \Gamma_{i}=\nu_{\theta^{\prime}} \alpha \int_{\Gamma_{i}}\left(\frac{g p}{R \bar{\theta}}\right)^{2} \theta^{\prime} \theta_{*}^{\prime} d \Gamma_{i} \\
& \quad+\left(-\frac{\theta_{h} N_{h}^{2}}{g} \omega-\frac{L}{c_{p} \Pi}\left(F \mathcal{H}_{\varepsilon_{2}}\left(q_{v}-q_{v s}\right)+k_{3} \tau\left(q_{r}\right)^{0.5}\left(q_{v s}-q_{v}\right)^{+}\right)+f_{\theta}^{1}+\omega \frac{\partial \theta_{h}(p)}{\partial p}, \theta^{\prime}\right) \\
& \quad \leqslant \frac{\nu_{\theta^{\prime}} \alpha}{2} \int_{\Gamma_{i}}\left(\frac{g p}{R \bar{\theta}}\right)^{2} \theta^{\prime 2} d \Gamma_{i}+\frac{\nu_{\theta^{\prime}} \alpha}{2} \int_{\Gamma_{i}}\left(\frac{g p}{R \bar{\theta}}\right)^{2} \theta_{*}^{\prime 2} d \Gamma_{i}+\kappa_{1}\left|\theta^{\prime}\right|_{L^{2}}^{2}+\kappa_{2} .
\end{aligned}
$$

Indeed, it is easy to see that the second term in the middle equation (4.57) is bounded by $\kappa_{1}\left|\theta^{\prime}\right|_{L^{2}}^{2}+\kappa_{2}$ since the terms $\theta_{h}, \omega$ and $\partial \theta_{h}(p) / \partial p$ are bounded in $L^{\infty}(\mathcal{M})$. In particular, (4.57) yields

$$
\frac{1}{2} \frac{d}{d t}\left|\theta^{\prime}\right|_{L^{2}}^{2}+\mu_{\theta^{\prime}}\left|\nabla \theta^{\prime}\right|_{L^{2}}^{2}+\nu_{\theta^{\prime}}\left|\frac{g p}{R \bar{\theta}} \frac{\partial \theta^{\prime}}{\partial p}\right|_{L^{2}}^{2} \leqslant \kappa_{1}\left|\theta^{\prime}\right|_{L^{2}}^{2}+\kappa_{2} .
$$

As before, the application of the Gronwall Lemma to (4.58) gives the following estimates

$$
\begin{aligned}
& \left|\theta^{\prime} \varepsilon\right|_{L^{\infty}\left(0, t_{1} ; L^{2}(\mathcal{M})\right)} \leqslant \kappa, \\
& \left|\theta^{\prime}\right|_{L^{2}\left(0, t_{1} ; H^{1}(\mathcal{M})\right)} \leqslant \kappa .
\end{aligned}
$$

Finally, for the $q_{r}$-equation given by $(4.33)_{3}$, we write the equivalent of $(4.44)$, which is simply obtained by multiplying $(4.33)_{3}$ by $q_{r}$ and integrating over $\mathcal{M}$, and we use (3.19). Therefore, using the fact that $\tau\left(q_{r}\right)$ and $\partial \theta^{\prime} / \partial p$ are bounded independently of $\varepsilon$ in $L^{2}(\mathcal{M})$ for a.e. $t \geqslant 0$, see the definition of $\tau\left(q_{r}\right)$ in the end of Remark ??, we obtain, 
with $\theta_{\wedge \alpha}=\min (\theta, \alpha)$, see after (3.19):

$$
\begin{aligned}
& \frac{1}{2} \frac{d}{d t}\left|q_{r}\right|_{L^{2}}^{2}+\mu_{q_{r}}\left|\nabla q_{r}\right|_{L^{2}}^{2}+\nu_{q_{r}}\left|\frac{g p}{R \bar{\theta}} \frac{\partial q_{r}}{\partial p}\right|_{L^{2}}^{2}+\nu_{q_{r}} \beta_{r} \int_{\Gamma_{i}}\left(\frac{g p}{R \bar{\theta}}\right)^{2} q_{r}^{2} d \Gamma_{i} \\
& \quad=\nu_{q_{r}} \beta_{r} \int_{\Gamma_{i}}\left(\frac{g p}{R \bar{\theta}}\right)^{2} q_{r} q_{r *} d \Gamma_{i}+\left(-5.32 g\left(\frac{\tau\left(q_{r}\right)^{1.2}}{R \Pi \theta_{\alpha}}+\frac{1.2 p \tau\left(q_{r}\right)^{0.2}}{R \Pi \theta_{\alpha}} \frac{\partial q_{r}}{\partial p}\right.\right. \\
& \left.\quad-\frac{p \tau\left(q_{r}\right)^{1.2}}{R \Pi \theta_{\alpha}^{2}}\left(\theta_{\alpha} \frac{\kappa}{p}+\frac{\partial \theta_{h}(p)}{\partial p}+\frac{\partial \theta^{\prime}}{\partial p}\right)\right) \\
& \left.\quad-k_{3} \tau\left(q_{r}\right)^{0.5}\left(q_{v s}-q_{v}\right)^{+}+k_{1}\left(q_{c}-q_{c r i t}\right)^{+}+k_{2} q_{c} \tau\left(q_{r}\right)^{0.875}, q_{r}\right) \\
& \quad \leqslant\left(\text { using the fact that } 0 \leqslant \tau\left(q_{r}\right) \leqslant 1 \text { and } \theta_{\alpha} \leqslant \alpha\right) \\
& \quad \leqslant \frac{\nu_{q_{r}} \beta_{r}}{2} \int_{\Gamma_{i}}\left(\frac{g p}{R \bar{\theta}}\right)^{2} q_{r}^{2} d \Gamma_{i}+\frac{\nu_{q_{r}} \beta_{r}}{2} \int_{\Gamma_{i}}\left(\frac{g p}{R \bar{\theta}}\right)^{2} q_{r *} d \Gamma_{i}+\beta\left|q_{v}\right|_{L^{2}}\left|q_{r}\right|_{L^{2}}+k_{1}\left|q_{c}\right| L_{L^{2}}\left|q_{r}\right|_{L^{2}} \\
& \quad+\kappa_{1}\left|q_{r}\right|_{L^{2}}^{2}+\kappa_{2}+\kappa_{1} \int_{\mathcal{M}}\left\{\left|\frac{\partial q_{r}}{\partial p}\right|+\left|\frac{\partial \theta^{\prime}}{\partial p}\right|+\left|q_{c}\right|\right\} d \mathcal{M} .
\end{aligned}
$$

As mentioned above, $q_{c}, q_{v}$ and $\partial \theta^{\prime} / \partial p$ are bounded in $L^{2}\left(0, t_{1} ; L^{2}(\mathcal{M})\right)$ independently of $\varepsilon$. Hence we infer that

$$
\frac{1}{2} \frac{d}{d t}\left|q_{r}\right|_{L^{2}}^{2}+\mu_{q_{r}}\left|\nabla q_{r}\right|_{L^{2}}^{2}+\nu_{q_{r}}\left|\frac{g p}{R \bar{\theta}} \frac{\partial q_{r}}{\partial p}\right|_{L^{2}}^{2} \leqslant \mathcal{G}(t),
$$

where $\mathcal{G}=\mathcal{G}(t)$ is a generic function of $t$, bounded in $L^{1}\left(0, t_{1}\right)$ independently of $\varepsilon$.

Finally it suffices to apply the Gronwall inequality to conclude that,

$$
\begin{aligned}
& \left|q_{r}^{\varepsilon}\right|_{L^{\infty}\left(0, t_{1} ; L^{2}(\mathcal{M})\right)} \leqslant \kappa, \\
& \left|q_{r}^{\varepsilon}\right|_{L^{2}\left(0, t_{1} ; H^{1}(\mathcal{M})\right)} \leqslant \kappa .
\end{aligned}
$$

4.5. A priori estimates on the time derivative of $U$. We now aim to derive a priori estimates for the time derivatives of $U$ in view of obtaining a strong convergence result for these functions and especially $\theta^{\prime}(\sim T)$, by application of a compactness theorem.

More precisely, we prove in this subsection some a priori estimates for the solution $U$ of the system (4.33) associated with the initial and boundary conditions (4.34) and (4.35), respectively. The intent is to show that the time derivative of $\bar{U}=\bar{U}^{\varepsilon}=\left(q_{c}^{\varepsilon}, q_{r}^{\varepsilon}, \theta^{\prime} \varepsilon\right)$ and $q_{v}^{\varepsilon}$, recalling here the dependence of the solution $U$ on $\varepsilon$, are bounded independently of $\varepsilon$. Therefore, for $\bar{U}^{\varepsilon}$, as we did in Subsection 4.4 we will develop here the computations for one component of $\bar{U}$ and then explain only the differences for the other components. The estimate for the time derivative of $q_{v}^{\varepsilon}$ is more subtle and will be treated differently later on (see a similar easier situation in [TWu15], [TWa15]). More precisely, let us start with the $q_{c}$-equation $(4.33)_{2}$ that we multiply by $\partial q_{c} / \partial t$ and integrate over $\mathcal{M}$. 
Hence, using the symmetry of $\mathcal{A}_{c}$ and then the Cauchy-Schwarz inequality, we obtain

$$
\begin{aligned}
\int_{\mathcal{M}}\left|\frac{\partial q_{c}}{\partial t}\right|^{2} d \mathcal{M}+\frac{1}{2} \frac{d}{d t}\left(\mathcal{A}_{c} q_{c}, q_{c}\right) & =\left(f_{q_{c}}(U)-F \mathcal{H}_{\varepsilon_{2}}\left(q_{v}-q_{v s}\right)-\mathbf{v} \cdot \nabla q_{c}-\omega \frac{\partial q_{c}}{\partial p}, \frac{\partial q_{c}}{\partial t}\right) \\
& \leqslant J_{1}+\frac{1}{2}\left|\frac{\partial q_{c}}{\partial t}\right|_{L^{2}}^{2},
\end{aligned}
$$

where

$$
J_{1}=c\left|f_{q_{c}}(U)-F \mathcal{H}_{\varepsilon_{2}}\left(q_{v}-q_{v s}\right)-\mathbf{v} \cdot \nabla q_{c}-\omega \frac{\partial q_{c}}{\partial p}\right|_{L^{2}}^{2}
$$

for an appropriate constant $c$.

To bound the term $J_{1}$ in the RHS of (4.65), we need first to estimate $\left|f_{q_{c}}(U)\right|_{L^{2}}$. Let us recall the definition of $f_{q_{c}}(U)$, which is given by (3.11) and it involves itself the expression of $\tau\left(q_{r}\right)$ stated just after (3.11). Hence, we estimate the terms in the RHS of (3.11), one by one, as follows:

$$
\begin{aligned}
\left|k_{1}\left(q_{c}-q_{c r i t}\right)^{+}\right| & \leqslant \kappa\left(1+\left|q_{c}\right|_{L^{2}}\right), \\
\left|k_{2} q_{c} \tau\left(q_{r}\right)\right| & \leqslant \kappa .
\end{aligned}
$$

Therefore, using the fact that $q_{v s}$ and $\tau\left(q_{r}\right)$ are bounded in $L^{\infty}\left(\mathcal{M} \times\left(0, t_{1}\right)\right)$ and $q_{c}$ is bounded in $L^{\infty}\left(0, t_{1} ; L^{2}(\mathcal{M})\right)$, we deduce that

$$
\left|f_{q_{c}}(U)\right|_{L^{2}} \leqslant \kappa\left(1+\left|q_{c}\right|_{L^{2}}\right) \leqslant \kappa, \quad \forall t \in\left(0, t_{1}\right) .
$$

Secondly, as $F$ and $\mathcal{H}_{\varepsilon_{2}}\left(q_{v}-q_{v s}\right)$ are bounded in $L^{\infty}\left(\mathcal{M} \times\left(0, t_{1}\right)\right)$, we also have

$$
\left|F \mathcal{H}_{\varepsilon_{2}}\left(q_{v}-q_{v s}\right)\right|_{L^{2}} \leqslant \kappa, \quad \forall t \in\left(0, t_{1}\right) .
$$

Then, assuming that $\mathbf{u} \in L^{\infty}\left(\mathcal{M} \times\left(0, t_{1}\right)\right)$ and using (4.50), we obtain

$$
\left|\mathbf{v} \cdot \nabla q_{c}\right|_{L^{2}} \leqslant|\mathbf{v}|_{L^{\infty}(\mathcal{M} \times(0, T))}\left|\nabla q_{c}\right|_{L^{2}} \leqslant \mathcal{G}(t),
$$

where we denoted again by $\mathcal{G}=\mathcal{G}(t)$ a generic function of time $t$, bounded in $L^{1}\left(0, t_{1}\right)$ independently of $\varepsilon$ and we recall that $\left|\nabla q_{c}\right|_{L^{2}}^{2}$ has been already bounded in $L^{1}\left(0, t_{1}\right)$. Using again $\mathbf{u} \in L^{\infty}\left(\mathcal{M} \times\left(0, t_{1}\right)\right)$ and $(4.50)$, we infer that

$$
\left|\omega \frac{\partial q_{c}}{\partial p}\right|_{L^{2}} \leqslant \kappa\left|\frac{\partial q_{c}}{\partial p}\right|_{L^{2}} \leqslant \mathcal{G}(t)
$$

and we recall that $\left|\partial q_{c} / \partial p\right|_{L^{2}}^{2}$ has been by now bounded in $L^{1}\left(0, t_{1}\right)$.

Now we derive similar estimates for the other terms $q_{r}$ and $\theta^{\prime}$. For that purpose, we follow the same steps as we did for $q_{c}$, and we write the equations analogous to (4.65). Hence, the only difference will be here the estimates of the terms $\left\|f_{q_{r}}(U)\right\|_{L^{2}}$ and $\left\|f_{\theta^{\prime}}(U)-\frac{L}{c_{p} \Pi} F \mathcal{H}_{\varepsilon_{2}}\left(q_{v}-q_{v s}\right)\right\|_{L^{2}}$. To do that we make use of the expressions of $f_{q_{r}}(U)$ and $f_{\theta^{\prime}}(U)-\frac{L}{c_{p} \Pi} F \mathcal{H}_{\varepsilon_{2}}\left(q_{v}-q_{v s}\right)$, given respectively by (3.19) and (3.23). For $f_{q_{r}}(U)$, we 
have

$$
\begin{aligned}
& \left|f_{q_{r}}(U)\right|_{L^{2}}= \\
& \mid-5.32 g\left(\frac{\tau\left(q_{r}\right)^{1.2}}{R \Pi \theta_{\alpha}}+\frac{1.2 p \tau\left(q_{r}\right)^{0.2}}{R \Pi \theta_{\alpha}} \frac{\partial q_{r}}{\partial p}-\frac{p \tau\left(q_{r}\right)^{1.2}}{R \Pi \theta_{\alpha}^{2}}\left(\theta_{\alpha} \frac{\kappa}{p}+\frac{\partial \theta_{h}(p)}{\partial p}+\frac{\partial \theta^{\prime}}{\partial p}\right)\right) \\
& -k_{3} \tau\left(q_{r}\right)^{0.5}\left(q_{v s}-q_{v}\right)^{+}+k_{1}\left(q_{c}-q_{c r i t}\right)^{+}+\left.k_{2} q_{c} \tau\left(q_{r}\right)^{0.875}\right|_{L^{2}} \\
& \leqslant \kappa_{1}\left\{\left|\frac{\partial q_{r}}{\partial p}\right|_{L^{2}}+\left|\frac{\partial \theta^{\prime}}{\partial p}\right|_{L^{2}}+\left|q_{c}\right|_{L^{2}}+\left|q_{v}\right|_{L^{2}}\right\}+\kappa_{2} \\
& \leqslant \mathcal{G}(t)+\left|\frac{\partial q_{r}}{\partial p}\right|_{L^{2}}^{2}+\left|\frac{\partial \theta^{\prime}}{\partial p}\right|_{L^{2}}^{2}+\left|q_{c}\right|_{L^{2}}^{2}+\left|q_{v}\right|_{L^{2}}^{2},
\end{aligned}
$$

and we already showed that $\left|\partial q_{r} / \partial p\right|_{L^{2}}^{2},\left|\partial \theta^{\prime} / \partial p\right|_{L^{2}}^{2},\left|q_{c}\right|_{L^{2}}^{2}$, and $\left|q_{v}\right|_{L^{2}}^{2}$ are bounded in $L^{1}\left(0, t_{1}\right)$ thanks to $(4.64),(4.60),(4.49)$ and $(4.54)$.

Similarly, for $f_{\theta^{\prime}}(U)-\frac{L}{c_{p} \Pi} F \mathcal{H}_{\varepsilon_{2}}\left(q_{v}-q_{v s}\right)$, we assume that $\mathbf{u} \in L^{\infty}\left(\mathcal{M} \times\left(0, t_{1}\right)\right)$ and we obtain

$$
\left|f_{\theta^{\prime}}(U)-\frac{L}{c_{p} \Pi} F \mathcal{H}_{\varepsilon_{2}}\left(q_{v}-q_{v s}\right)\right|_{L^{2}} \leqslant \mathcal{G}(t)+\left|q_{v}\right|_{L^{2}}^{2}
$$

Therefore we conclude that $J_{1} \leqslant \mathcal{G}(t)$ for all $t \in\left(0, t_{1}\right)$, and we infer that

$$
\left|\partial_{t} q_{c}^{\varepsilon}\right|_{L^{2}\left(0, t_{1} ; L^{2}(\mathcal{M})\right)} \leqslant \kappa
$$

here $\kappa$ is a constant independent of $\varepsilon$ and $t$.

Now we take the $L^{2}(\mathcal{M})$ inner product of $\mathcal{A}_{c} q_{c}$ with $(4.33)_{2}$, and we can apply a similar argument as what we did for $\partial_{t} q_{c}$ and obtain

$$
\left|\mathcal{A}_{c} q_{c}\right|_{L^{2}\left(0, t_{1} ; L^{2}\right)} \leqslant \kappa \text {. }
$$

Consequently, using (4.50), (4.75), (4.76) and (4.33) 2 , we deduce that

$$
\frac{\partial q_{c}^{\varepsilon}}{\partial t} \text { and } \mathcal{A}_{c} q_{c}^{\varepsilon} \text { are bounded in } L^{2}\left(0, t_{1} ; L^{2}\right) \text {, independently of } \varepsilon,
$$

that is $q_{c}$ is bounded in $L^{2}\left(0, t_{1} ; H^{2}\right)$ independently of $\varepsilon$.

Similar estimates to (4.75) follow in a straightforward manner for $q_{r}$ and $\theta^{\prime}$.

Before we move on to bound the time derivative of $q_{v}$, we add one more estimate on $\bar{U}$. Integrating $(4.65)$ on $(0, t)$ for any $t \in\left[0, t_{1}\right]$, we have

$$
\frac{1}{2} \int_{0}^{t}\left|\frac{\partial q_{c}}{\partial t}\right|_{L^{2}}^{2} d t+\left(\mathcal{A}_{c} q_{c}(t), q_{c}(t)\right) \leqslant \int_{0}^{t_{1}} J_{1} d t+\left(\mathcal{A}_{c} q_{c 0}, q_{c 0}\right) \leqslant \kappa
$$

where $J_{1}$ was defined in (4.66). Again, the constant $\kappa$ is independent of $\varepsilon$ and $t$. So $\left(\mathcal{A}_{c} q_{c}(t), q_{c}(t)\right)$ is bounded uniformly in time for any $t \in\left[0, t_{1}\right]$. This implies

$$
\left|q_{c}^{\varepsilon}\right|_{L^{\infty}\left(0, t_{1} ; H^{1}(\mathcal{M})\right)} \leqslant \kappa .
$$


Estimates similar to (4.79) hold for $q_{r}$ and $\theta^{\prime}$. In particular, we will use the bound

$$
\left|\theta^{\prime \varepsilon}\right|_{L^{\infty}\left(0, t_{1} ; H^{1}(\mathcal{M})\right)} \leqslant \kappa
$$

in the estimate of the time derivative of $q_{v}^{\varepsilon}$.

For $q_{v}^{\varepsilon}$, we will show its time derivative is bounded independently of $\varepsilon$ in $L^{5 / 3}\left(0, t_{1} ; V^{*}\right)$. The main issue here is to control the penalization term which contains the "large" factor $\frac{1}{\varepsilon_{1}}$. We begin with Lemma 4.2 .

Lemma 4.2. The following bound holds:

$$
\frac{1}{\varepsilon_{1}^{5 / 3}} \int_{0}^{t_{1}}\left|\left(q_{v}^{\varepsilon}-q_{v s}^{\varepsilon}\right)^{+}\right|_{L^{5 / 2}(\mathcal{M})}^{5 / 2} d t \leqslant \kappa,
$$

where $\kappa$ is a constant independent of $\varepsilon$.

Proof. We multiply $(4.33)_{1}$ by $\left(q_{v}-q_{v s}\right)^{+}$and integrate on $\mathcal{M}$, we find

$$
\begin{aligned}
& \left(\frac{\partial q_{v}}{\partial t},\left(q_{v}-q_{v s}\right)^{+}\right)+\left(\mathcal{A}_{v} q_{v},\left(q_{v}-q_{v s}\right)^{+}\right)+\frac{1}{\varepsilon_{1}} \int_{\mathcal{M}}\left(\left(q_{v}-q_{v s}\right)+\right)^{5 / 2} d \mathcal{M} \\
& =\left(f_{q_{v}}(U)+F \mathcal{H}_{\varepsilon_{2}}\left(q_{v}-q_{v s}\right)-\mathbf{v} \cdot \nabla q_{v}-\omega \frac{\partial q_{v}}{\partial p},\left(q_{v}-q_{v s}\right)^{+}\right) .
\end{aligned}
$$

The first two terms in the LHS can be rewritten as

$$
\begin{aligned}
\left(\partial_{t} q_{v},\left(q_{v}-q_{v s}\right)^{+}\right) & =\left(\partial_{t}\left(q_{v}-q_{v s}\right),\left(q_{v}-q_{v s}\right)^{+}\right)+\left(\partial_{t} q_{v s},\left(q_{v}-q_{v s}\right)^{+}\right) \\
& =\frac{1}{2} \frac{d}{d t}\left|\left(q_{v}-q_{v s}\right)^{+}\right|_{L^{2}(\mathcal{M})}^{2}+\left(\partial_{t} q_{v s},\left(q_{v}-q_{v s}\right)^{+}\right) \\
\left(\mathcal{A}_{v} q_{v},\left(q_{v}-q_{v s}\right)^{+}\right) & =\left(\mathcal{A}_{v}\left(q_{v}-q_{v s}\right),\left(q_{v}-q_{v s}\right)^{+}\right)+\left(\mathcal{A}_{v} q_{v s},\left(q_{v}-q_{v s}\right)^{+}\right) \\
& =\left(\mathcal{A}_{v}\left(q_{v}-q_{v s}\right)^{+},\left(q_{v}-q_{v s}\right)^{+}\right)+\left(\mathcal{A}_{v} q_{v s},\left(q_{v}-q_{v s}\right)^{+}\right) .
\end{aligned}
$$

Dropping the positive term: $\left(\mathcal{A}_{v}\left(q_{v}-q_{v s}\right)^{+},\left(q_{v}-q_{v s}\right)^{+}\right)$in the LHS, we can deduce from (4.82) that

$$
\begin{aligned}
& \frac{1}{2} \frac{d}{d t}\left|\left(q_{v}-q_{v s}\right)^{+}\right|_{L^{2}}^{2}+\frac{1}{\varepsilon_{1}}\left|\left(\left(q_{v}-q_{v s}\right)+\right)\right|_{L^{5 / 2}}^{5 / 2} \\
& \leqslant \mid\left(\mathcal{A}_{v} q_{v s},\left(q_{v}-q_{v s}\right)^{+}\right)+\left(\partial_{t} q_{v s},\left(q_{v}-q_{v s}\right)^{+}\right)+\left(\mathbf{u} \cdot \nabla_{3} q_{v},\left(q_{v}-q_{v s}\right)^{+}\right) \\
& \left.-\left(f_{q_{v}}(U)+F \mathcal{H}_{\varepsilon_{2}}\left(q_{v}-q_{v s}\right)\right),\left(q_{v}-q_{v s}\right)^{+}\right) \mid .
\end{aligned}
$$

Using Hölder and Young inequalities, the RHS of (4.83) can be estimated in the following 
way:

$$
\begin{aligned}
\left|\left(\mathcal{A}_{v} q_{v s},\left(q_{v}-q_{v s}\right)^{+}\right)\right| & =\left|\int_{\mathcal{M}} \varepsilon_{1}^{2 / 5} \mathcal{A}_{v} q_{v s} \cdot \frac{\left(q_{v}-q_{v s}\right)^{+}}{\varepsilon_{1}^{2 / 5}} d \mathcal{M}\right| \\
& \leqslant\left|\varepsilon_{1}^{2 / 5} \mathcal{A}_{v} q_{v s}\right|_{L^{5 / 3}}\left|\frac{\left(q_{v}-q_{v s}\right)^{+}}{\varepsilon_{1}^{2 / 5}}\right|_{L^{5 / 2}} \\
& \leqslant C \varepsilon_{1}^{2 / 3}\left|\mathcal{A}_{v} q_{v s}\right|_{L^{5 / 3}}^{5 / 3}+\frac{1}{8 \varepsilon_{1}}\left|\left(q_{v}-q_{v s}\right)^{+}\right|_{L^{5 / 2}}^{5 / 2} .
\end{aligned}
$$

The other terms can be addressed similarly. Then (4.83) becomes

$$
\begin{aligned}
& \frac{1}{2} \frac{d}{d t}\left|\left(q_{v}-q_{v s}\right)^{+}\right|_{L^{2}}^{2}+\frac{1}{\varepsilon_{1}}\left|\left(q_{v}-q_{v s}\right)^{+}\right|_{L^{5 / 2}}^{5 / 2} \\
& \leqslant C \varepsilon_{1}^{2 / 3}\left(\left|\mathcal{A}_{v} q_{v s}\right|_{L^{5 / 3}}^{5 / 3}+\left|\partial_{t} q_{v s}\right|_{L^{5 / 3}}^{5 / 3}+\left|\nabla_{3} q_{v}\right|_{L^{5 / 3}}^{5 / 3}+\left|q_{v}\right|_{L^{5 / 3}}^{5 / 3}\right. \\
& \left.+C_{1}\right)+\frac{1}{2 \varepsilon_{1}}\left|\left(q_{v}-q_{v s}\right)^{+}\right|_{L^{5 / 2}}^{5 / 2} .
\end{aligned}
$$

Here $\left|q_{v}\right|_{L^{5 / 3}}^{5 / 3}+C_{1}$ in the RHS of (4.85) is the bound for $\left|f_{q_{v}}(U)+F \mathcal{H}_{\varepsilon_{2}}\left(q_{v}-q_{v s}\right)\right|_{L^{5 / 3}}^{5 / 3}$.

Integrating now (4.85) in time on $\left(0, t_{1}\right)$, we have

$$
\begin{aligned}
& \frac{1}{2}\left|\left(q_{v}\left(t_{1}\right)-q_{v s}\left(t_{1}\right)\right)^{+}\right|_{L^{2}}^{2}-\frac{1}{2}\left|\left(q_{v 0}-q_{s 0}\right)^{+}\right|_{L^{2}}^{2}+\frac{1}{2 \varepsilon_{1}} \int_{0}^{t_{1}}\left|\left(q_{v}-q_{v s}\right)^{+}\right|_{L^{5 / 2}}^{5 / 2} d t \\
& \leqslant C \varepsilon_{1}^{2 / 3} \int_{0}^{t_{1}}\left(\left|\mathcal{A}_{v} q_{v s}\right|_{L^{5 / 3}}^{5 / 3}+\left|\partial_{t} q_{v s}\right|_{L^{5 / 3}}^{5 / 3}+\left|\nabla_{3} q_{v}\right|_{L^{5 / 3}(\mathcal{M})}^{5 / 3}+\left|q_{v}\right|_{L^{5 / 3}}^{5 / 3}+C_{1}\right) d t .
\end{aligned}
$$

The first term in the LHS of (4.86) is positive and the second term is 0 because of the constraint on the initial value $q_{v 0} \leqslant q_{s 0}$.

To reach the desired bound (4.81) on the penalization term, we will bound the integral in the RHS of (4.86) independently of $\varepsilon$, drop the positive term in the LHS and divide both sides of (4.86) by $\varepsilon_{1}^{2 / 3}$. We now estimate each term in the RHS of (4.86).

Both $\left|q_{v}\right|$ and $\left|\nabla_{3} q_{v}\right|$ are bounded in $L^{5 / 3}\left(\left(0, t_{1}\right) \times \mathcal{M}\right)$, thanks to (4.54),(4.55) and the fact that $L^{2}\left(\left(0, t_{1}\right) \times \mathcal{M}\right) \subset L^{5 / 3}\left(\left(0, t_{1}\right) \times \mathcal{M}\right)$.

Then for $\partial_{t} q_{v s}$, we see that $\partial_{t} q_{v s}=\frac{\partial Q_{v s}}{\partial T}(p, T) \cdot \partial_{t} T$. Because of the relationship between $T$ (resp. $T^{\prime}$ ) and $\theta$ (resp. $\theta^{\prime}$ ) and recalling that $\partial_{t} \theta^{\prime}$ has already been bounded in $L^{2}\left(\left(0, t_{1}\right) \times \mathcal{M}\right), \partial_{t} T$ is bounded in $L^{2}\left(\left(0, t_{1}\right) \times \mathcal{M}\right)$. Also, $\frac{\partial Q_{v s}}{\partial T}(p, T)$ is uniformly bounded by Remark 2.1. Thus we have $\left|\partial_{t} q_{v s}\right|$ bounded in $L^{5 / 3}\left(\left(0, t_{1}\right) \times \mathcal{M}\right)$.

The most problematic term is $\left|\mathcal{A}_{v} q_{v s}\right|_{L^{5 / 3}}^{5 / 3}$. We begin by exploring the relationship between $\Delta_{3} q_{v s}$ and $T$. By the expressions (2.7) and (2.8), 


$$
\begin{aligned}
\frac{\partial^{2} q_{v s}}{\partial x^{2}} & =\frac{\partial}{\partial x}\left(\frac{\partial Q_{v s}(p, T)}{\partial T} \frac{\partial T}{\partial x}\right) \\
& =\frac{\partial}{\partial x}\left(\frac{\partial Q_{v s}(p, T)}{\partial T}\right) \frac{\partial T}{\partial x}+\frac{\partial Q_{v s}(p, T)}{\partial T} \frac{\partial^{2} T}{\partial x^{2}} .
\end{aligned}
$$

Use the fact that $\partial q_{v s}(p, T) / \partial T$ is uniformly bounded as stated in Remark 2.1, we can easily deduce that

$$
\left|\frac{\partial}{\partial x}\left(\frac{\partial Q_{v s}(p, T)}{\partial T}\right)\right| \leqslant C\left|\frac{\partial T}{\partial x}\right|
$$

for some generic constant $\mathrm{C}$ that does not depend on $\varepsilon$.

Recalling (4.87), we can further deduce that, pointwisely,

$$
\left|\frac{\partial^{2} q_{v s}}{\partial x^{2}}\right| \leqslant C\left(\left|\frac{\partial T}{\partial x}\right|^{2}+\left|\frac{\partial^{2} T}{\partial x^{2}}\right|\right)
$$

Similarly,

$$
\left|\frac{\partial^{2} q_{v s}}{\partial y^{2}}\right| \leqslant C\left(\left|\frac{\partial T}{\partial y}\right|^{2}+\left|\frac{\partial^{2} T}{\partial y^{2}}\right|\right)
$$

The second derivative of $q_{v s}$ with respect to $\mathrm{p}$ is slightly different with $\partial^{2} q_{v s} / \partial x^{2}$ and $\partial^{2} q_{v s} / \partial y^{2}$, as $q_{v s}$ depends on $p$ explicitly.

By (2.8), it can be easily calculated that

$$
\frac{\partial q_{v s}}{\partial p}=\frac{1}{\left(p-0.378 e_{v s}\right)}\left(\frac{273 a p}{T^{2}} \cdot \frac{\partial T}{\partial p}-1\right) q_{v s} .
$$

Differentiating with respect to p one more time, we can deduce that, after some algebra:

$$
\left|\frac{\partial^{2} q_{v s}}{\partial p^{2}}\right| \leqslant C\left(\left|\frac{\partial T}{\partial p}\right|+\left|\frac{\partial T}{\partial p}\right|^{2}+\left|\frac{\partial^{2} T}{\partial p^{2}}\right|+C_{1}\right) .
$$

It follows that

$$
\left|\mathcal{A}_{v} q_{v s}\right|_{L^{5 / 3}(\mathcal{M})}^{5 / 3} \leqslant C\left(\left|\Delta_{3} T\right|_{L^{5 / 3}(\mathcal{M})}^{5 / 3}+\left|\nabla_{3} T\right|_{L^{10 / 3}(\mathcal{M})}^{10 / 3}+C_{1}\right) .
$$

By Gagliardo-Nirenberg's interpolation inequality, we have

$$
\left|\nabla_{3} T\right|_{L^{10 / 3}(\mathcal{M})}^{10 / 3} \leqslant C\left(\left|\nabla_{3} T\right|_{L^{2}(\mathcal{M})}^{10 / 3}+\left|\nabla_{3} T\right|_{L^{2}(\mathcal{M})}^{4 / 3}\left|\Delta_{3} T\right|_{L^{2}(\mathcal{M})}^{2}\right) .
$$


Note that we also have $\nabla_{3} T \in L^{\infty}\left(0, t_{1} ; H\right)$ and $\Delta_{3} T \in L^{2}\left(\left(0, t_{1}\right) \times \mathcal{M}\right)$ by $(4.76)$ and (4.80), and thus

$$
\begin{aligned}
\int_{0}^{t_{1}}\left|\mathcal{A}_{v} q_{v s}\right|_{L^{5 / 3}(\mathcal{M})}^{5 / 3} d t & \leqslant C \int_{0}^{t_{1}}\left(\left|\Delta_{3} T\right|_{L^{5 / 3}}^{5 / 3}+\left|\nabla_{3} T\right|_{L^{10 / 3}}^{10 / 3}+C_{1}\right) d t \\
& \leqslant C \int_{0}^{t_{1}}\left(\left|\Delta_{3} T\right|_{L^{5 / 3}}^{5 / 3}+\left|\nabla_{3} T\right|_{L^{2}}^{10 / 3}+\left|\nabla_{3} T\right|_{L^{2}}^{4 / 3}\left|\Delta_{3} T\right|_{L^{2}}^{2}+C_{1}\right) d t \\
& \leqslant C\left(\left|\Delta_{3} T\right|_{L^{5 / 3}\left(\left(0, t_{1}\right) \times \mathcal{M}\right)}^{5 / 3}+\underset{\left[0, t_{1}\right]}{\operatorname{ess} \sup }\left|\nabla_{3} T\right|_{L^{2}}\right)^{10 / 3} \cdot t_{1} \\
& \left.\left.+\underset{\left[0, t_{1}\right]}{\operatorname{ess} \sup }\left|\nabla_{3} T\right|_{L^{2}}\right)^{4 / 3}\left|\Delta_{3} T\right|_{L^{2}\left(\left(0, t_{1}\right) \times \mathcal{M}\right)}^{2}+C_{1} t_{1}\right) \\
& \leqslant \kappa
\end{aligned}
$$

By now all the terms in the integral in the RHS of (4.86) have been bounded independently of $\varepsilon$, this finishes the proof of Lemma 4.2.

With the help of Lemma 4.2, we are ready to estimate the time derivative of $q_{v}$. We multiply $(4.33)_{1}$ by $q_{v}^{b} \in L^{5 / 2}\left(0, t_{1} ; V\right)$ and integrate on $\mathcal{M}$ :

$$
\left\langle\partial_{t} q_{v}, q_{v}^{b}\right\rangle+\left(\mathcal{A}_{v} q_{v}, q_{v}^{b}\right)+\left(\mathbf{u} \cdot \nabla_{3} q_{v}, q_{v}^{b}\right)+\left(\frac{1}{\varepsilon_{1}}\left(\left(q_{v}-q_{v s}\right)^{+}\right)^{3 / 2}, q_{v}^{b}\right)=\left(f^{\varepsilon_{2}}(U), q_{v}^{b}\right) .
$$

Rearranging (4.91), we have

$$
\begin{aligned}
\left|\left\langle\partial_{t} q_{v}, q_{v}^{b}\right\rangle\right| & =\left|-a_{q_{v}}\left(q_{v}, q_{v}^{b}\right)-b\left(\mathbf{u}, q_{v}, q_{v}^{b}\right)-\left(\frac{1}{\varepsilon_{1}}\left(\left(q_{v}-q_{v s}\right)^{+}\right)^{3 / 2}, q_{v}^{b}\right)+l_{q_{v}}\left(q_{v}^{b}\right)+\left(f_{q_{v}}^{\varepsilon_{2}}, q_{v}^{b}\right)\right| \\
& \leqslant C\left(\left\|q_{v}\right\|_{V}+\|\mathbf{u}\|_{\mathbf{V}}\left\|q_{v}\right\|_{V}+\frac{1}{\varepsilon_{1}}\left|\left(q_{v}-q_{v s}\right)^{+}\right|_{L^{5 / 2}}^{3 / 2}+\left|q_{v}\right|_{L^{2}}+C_{1}\right)\left\|q_{v}^{b}\right\|_{V} .
\end{aligned}
$$

Here we used the Lemma 4.1 and the fact that

$$
\begin{aligned}
\frac{1}{\varepsilon_{1}} \int_{\mathcal{M}}\left(\left(q_{v}-q_{v s}\right)^{+}\right)^{3 / 2} q_{v}^{b} d \mathcal{M} & \leqslant \frac{1}{\varepsilon_{1}}\left|\left(\left(q_{v}-q_{v s}\right)^{+}\right)^{3 / 2}\right|_{L^{5 / 3}}\left|q_{v}^{b}\right|_{L^{5 / 2}} \\
& \leqslant\left(V \subset L^{5 / 2}(\mathcal{M}) \text { in } \mathbb{R}^{3}\right) \\
& \leqslant \frac{1}{\varepsilon_{1}}\left|\left(q_{v}-q_{v s}\right)^{+}\right|_{L^{5 / 2}}^{3 / 2}\left\|q_{v}^{b}\right\|_{V} .
\end{aligned}
$$

Hence,

$$
\begin{gathered}
\left\|\partial_{t} q_{v}\right\|_{V^{*}} \leqslant C\left(\left\|q_{v}\right\|_{V}+\|\mathbf{u}\|_{\mathbf{V}}\left\|q_{v}\right\|_{V}+\frac{1}{\varepsilon_{1}}\left|\left(q_{v}-q_{v s}\right)^{+}\right|_{L^{5 / 2}}^{3 / 2}+\left|q_{v}\right|_{L^{2}}+C_{1}\right), \\
\left\|\partial_{t} q_{v}\right\|_{V^{*}}^{5 / 3} \leqslant C\left(\left\|q_{v}\right\|_{V}^{5 / 3}+\|\mathbf{u}\|_{\mathbf{V}}^{5 / 3}\left\|q_{v}\right\|_{V}^{5 / 3}+\frac{1}{\varepsilon_{1}^{5 / 3}}\left|\left(q_{v}-q_{v s}\right)^{+}\right|_{L^{5 / 2}}^{5 / 2}+\left|q_{v}\right|_{L^{2}}^{5 / 3}+C_{1}\right) .
\end{gathered}
$$

Then thanks to (4.54),(4.55) and Lemma 4.2,

$$
\int_{0}^{t_{1}}\left\|\partial_{t} q_{v}\right\|_{V^{*}}^{5 / 3} d t \leqslant \kappa
$$

where $\kappa$, as before, is a constant independent of $\varepsilon$. So we have bound $\partial_{t} q_{v}$ in $L^{5 / 3}\left(0, t_{1} ; V^{*}\right)$ as desired. 
Finally, we summarize all the estimates that we obtained componentwise and write this for the solution $U^{\varepsilon}$. More precisely, we now have

$$
\begin{gathered}
\left|U^{\varepsilon}\right|_{L^{\infty}\left(0, t_{1} ; \mathbb{H}\right)} \leqslant \kappa,\left\|U^{\varepsilon}\right\|_{L^{2}\left(0, t_{1} ; \mathbb{V}\right)} \leqslant \kappa,\left\|\bar{U}^{\varepsilon}\right\|_{\left.L^{2}\left(0, t_{1} ; H^{2}(\mathcal{M})^{3}\right)\right)} \leqslant \kappa,\left\|\partial_{t} \bar{U}^{\varepsilon}\right\|_{L^{2}\left(0, t_{1} ; L^{2}(\mathcal{M})^{3}\right)} \leqslant \kappa, \\
\left\|\bar{U}^{\varepsilon}\right\|_{\left.L^{\infty}\left(0, t_{1} ; H^{1}(\mathcal{M})^{3}\right)\right)} \leqslant \kappa, \text { and }\left|\partial_{t} q_{v}^{\varepsilon}\right|_{L^{5 / 3}\left(0, t_{1} ; V^{*}\right)} \leqslant \kappa .
\end{gathered}
$$

Remark 4.3. As usual by implementing a Galerkin approximation for the problem (4.33)-(4.37) we can obtain a priori estimates similar to the above estimates for the Galerkin approximation. Then passing to the lower limit we obtain these very estimates (independent of $\varepsilon$ ) for the actual solution $U$ of (4.33)-(4.37). We state this existence result in the following theorem, but we will skip the proof since it is straightforward after the analysis above on the a priori estimates.

Theorem 4.4. Let $\varepsilon>0$ be fixed and assume that $\mathbf{u} \in L^{\infty}\left(\left(0, t_{1}\right) \times \mathcal{M}\right)$ and $U_{0} \in \mathbb{V}$ are given. Then, the system (4.33) associated with the initial and boundary conditions (4.34) and (4.35), respectively, has a solution $U^{\varepsilon}$ such that

$$
U^{\varepsilon} \in L^{\infty}\left(0, t_{1} ; \mathbb{H}\right) \cap L^{2}\left(0, t_{1} ; \mathbb{V}\right),
$$

and

$$
\bar{U}^{\varepsilon} \in L^{2}\left(0, t_{1} ; H^{2}\right), \quad \partial_{t} \bar{U}^{\varepsilon} \in L^{2}\left(0, t_{1} ; L^{2}\right), \quad \partial_{t} q_{v}^{\varepsilon} \in L^{5 / 3}\left(0, t_{1} ; V^{*}\right) .
$$

Furthermore the norms of $U^{\varepsilon}, \bar{U}^{\varepsilon}$ and $\partial_{t} \bar{U}^{\varepsilon}$ in the corresponding spaces are bounded independently of $\varepsilon$ by quantities which depend on the norm of $U_{0}$ in $\mathbb{H}$ and on the other data.

4.6. Passage to the limit. In the following we will pass to the limit, as $\varepsilon \rightarrow 0$, in the penalized system (4.33), and to avoid a possible confusion we reintroduce here the dependence on $\varepsilon$. First, using (4.97) and Aubin-Lions compactness theorem, we deduce the existence of a subsequence, still denoted $U^{\varepsilon}=\left(q_{v}^{\varepsilon}, q_{c}^{\varepsilon}, q_{r}^{\varepsilon}, \theta^{\prime} \varepsilon\right)$, and a function $U=\left(q_{v}, q_{c}, q_{r}, \theta^{\prime}\right)$ both verifying (4.98), (4.99), such that, as $\varepsilon \rightarrow 0$,

(i) $U^{\varepsilon} \rightarrow U$ weakly in $L^{2}\left(0, t_{1} ; \mathbb{V}\right)$ and weak-* in $L^{\infty}\left(0, t_{1} ; \mathbb{H}\right)$,

(ii) $\partial_{t} \bar{U}^{\varepsilon} \rightarrow \partial_{t} \bar{U}$ weakly in $L^{2}\left(0, t_{1} ; L^{2}(\mathcal{M})^{3}\right)$,

(iii) $\partial_{t} q_{v}^{\varepsilon} \rightarrow \partial_{t} q_{v}$ weakly in $L^{5 / 3}\left(0, t_{1} ; V^{*}\right)$,

(iv) $\bar{U}^{\varepsilon} \rightarrow \bar{U}$ strongly in $L^{2}\left(0, t_{1} ; H^{1}\right)$ and weakly in $L^{2}\left(0, t_{1} ; H^{2}\right)$,

(v) $q_{v}^{\varepsilon} \rightarrow q_{v}$ strongly in $L^{2}\left(0, t_{1} ; L^{2}(\mathcal{M})\right)$ and weakly in $L^{2}\left(0, t_{1} ; H^{1}\right)$,

(vi) $\left(q_{v}^{\varepsilon}-q_{v s}^{\varepsilon}\right)^{+} \rightarrow 0$ strongly in $L^{5 / 2}\left(\left(0, t_{1}\right) \times \mathcal{M}\right)$, thanks to Lemma 4.2 ,

(vii) $\mathcal{H}_{\varepsilon_{2}}\left(q_{v}^{\varepsilon}-q_{v s}^{\varepsilon}\right) \rightarrow h_{q_{v}}$ weak-* in $L^{\infty}\left(\left(0, t_{1}\right) \times \mathcal{M}\right)$ for $h_{q_{v}} \in \mathcal{H}\left(q_{v}-q_{v s}\right)$,

In view of (i) and (iii), we also have

$$
q_{v}^{\varepsilon}\left(t_{1}\right) \rightarrow q_{v}\left(t_{1}\right) \text { weakly in } L^{2}(\mathcal{M}) .
$$

For the inequality constraint on $q_{v}$, after showing that $q_{v s}^{\varepsilon} \rightarrow q_{v s}=Q_{v s}(p, T)$ in $L^{2}\left(0, t_{1} ; V\right)$ (see Lemma 4.5 below), (vi) implies in particular that $q_{v} \leqslant q_{v s}$.

It is worth noting here that the strong convergence in $L^{2}\left(0, t_{1} ; \mathbb{H}\right)$ is in fact available in $L^{p}\left(0, t_{1} ; \mathbb{H}\right)$, for all $p \geqslant 1$, thanks to the continuity of $U^{\varepsilon} \in \mathcal{C}\left(\left[0, t_{1}\right] ; \mathbb{H}\right)$. 
By the continuity and boundedness of $f(U)$, using (iv) and (v), we have

$$
f\left(U^{\varepsilon}\right) \rightarrow f(U) \text { strongly in } L^{2}\left(0, t_{1} ; L^{2}(\mathcal{M})\right) .
$$

where $f(U)$ is seen componentwise as $f(U)=\left(f_{q_{v}}(U), f_{q_{c}}(U), f_{q_{r}}(U), f_{\theta^{\prime}}(U)\right)$ and $\mathcal{F}$ represents the vector $\left(F,-F, 0,-\frac{L}{c_{p} \Pi} F\right)$.

Moreover, thanks to the estimates showing the boundedness of $\mathcal{F} \mathcal{H}_{\varepsilon_{2}}\left(q_{v}-q_{v s}\right)$ as performed above, using (vii) and [Lio76, Lemma 1.3], we have

$$
\mathcal{F}\left(p, T^{\varepsilon}\right) \mathcal{H}_{\varepsilon_{2}}\left(q_{v}-q_{v s}\right) \rightarrow \mathcal{F}(p, T) h_{q_{v}} \text { weakly in } L^{2}\left(0, t_{1} ; L^{2}(\mathcal{M})\right),
$$

Therefore one can pass to the limit, as $\varepsilon \rightarrow 0$, in (4.33) $2,3,4$ (remember here the dependence of the solutions on $\varepsilon$ ), see [TWa15] and [TWu15].

Moreover, we need the following results which will be used in the proof of the convergence of the penalized term, namely $(4.33)_{1}$.

Lemma 4.5. If $T^{\varepsilon}$ converges to $T$ strongly in $L^{2}\left(0, t_{1} ; V\right)$, then $q_{v s}^{\varepsilon}=Q_{v s}\left(p, T^{\varepsilon}\right)$, as given by $(2.7)$, converges to $q_{v s}=Q_{v s}(p, T)$ strongly in $L^{2}\left(0, t_{1} ; V\right)$.

Proof. By the expressions (2.7)-(2.9) and Remark 2.1, we see that $q_{v s}^{\varepsilon}=Q_{v s}\left(p, T^{\varepsilon}\right)$ converges to $q_{v s}=Q_{v s}(p, T)$ in $L^{2}\left(0, t_{1} ; L^{2}\right)$. Indeed, on the one hand, we recall here the relationship between $T$ (resp. $T^{\prime}$ and $T^{\prime} \varepsilon$ ) and $\theta$ (resp. $\theta^{\prime}$ and $\theta^{\prime} \varepsilon$ ) thanks to e.g. (2.12), and on the other hand, we use the fact that $\bar{U}^{\varepsilon}$ converges to $\bar{U}$ strongly in $\left(L^{2}\left(0, t_{1} ; V\right)\right)^{3}$. Similarly since the derivative of $Q_{v s}$ with respect to $T$ is uniformly bounded, thanks to 2.1 , we see that $\nabla_{\mathbf{x}} q_{v s}^{\varepsilon}=\frac{\partial Q_{v s}}{\partial T}\left(p, T^{\varepsilon}\right) \cdot \nabla_{\mathbf{x}} T^{\varepsilon}$ converges to $\nabla_{\mathbf{x}} q_{v s}=\frac{\partial Q_{v s}}{\partial T}(p, T) \cdot \nabla_{\mathbf{x}} T$ in $L^{2}\left(\left(0, t_{1}\right) \times \mathcal{M}\right)$.

We thus conclude that $q_{v s}^{\varepsilon}$ converges to $q_{v s}$ strongly in $L^{2}\left(0, t_{1} ; V\right)$.

Lemma 4.6. For all $q_{v}^{b} \in \mathcal{K}=\mathcal{K}(U)$, we consider $q_{v}^{b \varepsilon}=q_{v}^{b}-\left(q_{v}^{b}-q_{v s}^{\varepsilon}\right)^{+}=\min \left(q_{v}^{b}, q_{v s}^{\varepsilon}\right)$. Then $q_{v}^{b \varepsilon}$ converges to $q_{v}^{b}$ strongly in $L^{2}\left(0, t_{1} ; V\right)$.

Proof. We first observe, using the definitions of $q_{v}^{b \varepsilon}$ and of the set $\mathcal{K}$, that $q_{v}^{b \varepsilon}$ converges to $q_{v}^{b}$ in $L^{2}\left(0, t_{1} ; L^{2}\right)$. Then we see that the derivative of $q_{v}^{b \varepsilon}$ with respect to the space variable $\mathbf{x}$ can be written as $\nabla_{\mathbf{x}} q_{v}^{b \varepsilon}=\nabla_{\mathbf{x}} q_{v}^{b}-1_{\left\{q_{v}^{b}>q_{v s}^{\varepsilon}\right\}} \nabla_{\mathbf{x}}\left(q_{v}^{b}-q_{v s}^{\varepsilon}\right)$. Using Lemma 4.5 we deduce that $q_{v}^{b \varepsilon}$ converges to $q_{v}^{b}$ strongly in $L^{2}\left(0, t_{1} ; V\right)$.

Remark 4.7. From the proof of Lemma 4.6, we see that $\left|\nabla_{3} q_{v s}^{\varepsilon}\right| \leqslant C\left|\nabla_{3} T^{\varepsilon}\right|+C_{1}$. Then noting that $\nabla_{3} T^{\varepsilon} \in L^{\infty}\left(0, t_{1} ; L^{2}(\mathcal{M})\right)$ by $(4.80)$, here $q_{v s}^{\varepsilon}$ actually lies in a bounded set of $L^{\infty}\left(0, t_{1} ; V\right)$. And by our assumption, $q_{v}^{b \varepsilon} \in L^{\infty}\left(0, t_{1} ; V\right)$. Hence, $q_{v}^{b \varepsilon}=\min \left(q_{v}^{b}, q_{v s}^{\varepsilon}\right)$ lies in a bounded set in $L^{\infty}\left(0, t_{1} ; V\right)$ as well. Also $q_{v}^{b \varepsilon}$ converges to $q_{v}^{b}$ almost everywhere in $V$ for $t \in\left[0, t_{1}\right]$. Lemma 4.6 together with Lebesgue's dominated convergence theorem yields

$$
q_{v}^{b \varepsilon} \rightarrow q_{v}^{b} \text { strongly in } L^{p}\left(0, t_{1} ; V\right) \text { for any } p>1 .
$$

In particular, we will use the result with $p=\frac{5}{2}$ for passing to limit in the $q_{v}$-equation.

Now, for the $q_{v}$-equation $(4.33)_{1}$, the treatment will be different because of the penalization term as we will see below. Let us first rewrite as follows the weak formulation 
of the penalized equation $(4.33)_{1}$ in view of (4.28). For all $q_{v}^{b} \in \mathcal{K}(U)$, we consider $q_{v}^{b \varepsilon}=q_{v}^{b}-\left(q_{v}^{b}-q_{v s}^{\varepsilon}\right)^{+}=\min \left(q_{v}^{b}, q_{v s}^{\varepsilon}\right) \leqslant q_{v s}^{\varepsilon}$. We then write the first equation ( $q_{v}$-equation) of (4.36) with $q_{v}^{b}$ replaced by $q_{v}^{b \varepsilon}-q_{v}^{\varepsilon}$, and we find

$$
\begin{aligned}
\left\langle\partial_{t} q_{v}^{\varepsilon}, q_{v}^{b \varepsilon}-q_{v}^{\varepsilon}\right\rangle & +a_{q_{v}}\left(q_{v}^{\varepsilon}, q_{v}^{b \varepsilon}-q_{v}^{\varepsilon}\right)+b\left(\mathbf{u}, q_{v}^{\varepsilon}, q_{v}^{b \varepsilon}-q_{v}^{\varepsilon}\right)-l_{q_{v}}\left(q_{v}^{b \varepsilon}-q_{v}^{\varepsilon}\right) \\
& +\frac{1}{\varepsilon_{1}}\left\langle\left(\left(q_{v}^{\varepsilon}-q_{v s}^{\varepsilon}\right)^{+}\right)^{3 / 2}, q_{v}^{b \varepsilon}-q_{v}^{\varepsilon}\right\rangle=\left(f_{q_{v}}\left(U^{\varepsilon}\right)+F \mathcal{H}_{\varepsilon_{2}}\left(q_{v}-q_{v s}\right), q_{v}^{b \varepsilon}-q_{v}^{\varepsilon}\right) .
\end{aligned}
$$

Regarding (4.104), we first observe that

$$
\begin{aligned}
& \left\langle\left(\left(q_{v}^{\varepsilon}-q_{v s}^{\varepsilon}\right)^{+}\right)^{3 / 2}, q_{v}^{b \varepsilon}-q_{v}^{\varepsilon}\right\rangle \\
& =\langle\underbrace{\left(\left(q_{v}^{\varepsilon}-q_{v s}^{\varepsilon}\right)^{+}\right)^{3 / 2}, q_{v}^{b \varepsilon}-q_{v s}^{\varepsilon}}_{\left.\leqslant 0 \text { (because } q_{v}^{b \varepsilon} \leqslant q_{v s}^{\varepsilon}\right)}\rangle+\underbrace{\left\langle\left(\left(q_{v}^{\varepsilon}-q_{v s}^{\varepsilon}\right)^{+}\right)^{3 / 2},\left(q_{v s}^{\varepsilon}-q_{v}^{\varepsilon}\right)\right\rangle}_{\leqslant 0 \text { (by definition of the positive function) }} \leqslant 0 .
\end{aligned}
$$

Then, after integrating in time on $\left(0, t_{1}\right)$ and using (4.105), we rewrite (4.104) as follows:

$$
\begin{array}{r}
\int_{0}^{t_{1}}\left\langle\partial_{t} q_{v}^{\varepsilon}, q_{v}^{b \varepsilon}-q_{v}^{\varepsilon}\right\rangle d t+\int_{0}^{t_{1}} a_{q_{v}}\left(q_{v}^{\varepsilon}, q_{v}^{b \varepsilon}-q_{v}^{\varepsilon}\right) d t+\int_{0}^{t_{1}} b\left(\mathbf{u}, q_{v}^{\varepsilon}, q_{v}^{b \varepsilon}-q_{v}^{\varepsilon}\right) d t \\
-\int_{0}^{t_{1}} l_{q_{v}}\left(q_{v}^{b \varepsilon}-q_{v}^{\varepsilon}\right) d t \geqslant \int_{0}^{t_{1}}\left(f_{q_{v}}\left(U^{\varepsilon}\right)+F \mathcal{H}_{\varepsilon_{2}}\left(q_{v}-q_{v s}\right), q_{v}^{b \varepsilon}-q_{v}^{\varepsilon}\right) d t .
\end{array}
$$

In what follows we will justify the passage to the limit in (4.106), term by term. First, we observe that

$$
\begin{aligned}
& \int_{0}^{t_{1}}\left\langle\partial_{t} q_{v}^{\varepsilon},-q_{v}^{\varepsilon}\right\rangle d t=-\frac{1}{2} \int_{0}^{t_{1}} \frac{d}{d t}\left|q_{v}^{\varepsilon}\right|_{L^{2}}^{2}=-\frac{1}{2}\left|q_{v}^{\varepsilon}\left(t_{1}\right)\right|_{L^{2}}^{2}+\frac{1}{2}\left|q_{v 0}\right|_{L^{2}}^{2}, \\
& \limsup _{\varepsilon \rightarrow 0} \int_{0}^{t_{1}}\left\langle\partial_{t} q_{v}^{\varepsilon},-q_{v}^{\varepsilon}\right\rangle d t=-\liminf _{\varepsilon \rightarrow 0} \frac{1}{2}\left|q_{v}^{\varepsilon}\left(t_{1}\right)\right|_{L^{2}}^{2}+\frac{1}{2}\left|q_{v 0}\right|_{L^{2}}^{2} \\
& \leqslant-\frac{1}{2}\left|q_{v}\left(t_{1}\right)\right|_{L^{2}}^{2}+\frac{1}{2}\left|q_{v 0}\right|_{L^{2}}^{2} \\
& =-\int_{0}^{t_{1}}\left\langle\partial_{t} q_{v}, q_{v}\right\rangle d t
\end{aligned}
$$

In addition, by (iii), Lemma 4.6 and Remark 4.7, we have

$$
\left\langle\partial_{t} q_{v}^{\varepsilon}, q_{v}^{b \varepsilon}\right\rangle \rightarrow\left\langle\partial_{t} q_{v}, q_{v}^{b}\right\rangle \text {, as } \varepsilon \rightarrow 0 \text {. }
$$

We then obtain

$$
\limsup _{\varepsilon \rightarrow 0} \int_{0}^{t_{1}}\left\langle\partial_{t} q_{v}^{\varepsilon}, q_{v}^{b \varepsilon}-q_{v}^{\varepsilon}\right\rangle d t \leqslant \int_{0}^{t_{1}}\left\langle\partial_{t} q_{v}, q_{v}^{b}-q_{v}\right\rangle d t
$$

The convergence of the $a_{q_{v}}$-term in (4.106) is straightforward. Indeed, on the one hand, using Lemma 4.6 and the expression of $a$ as in (4.7), we have

$$
\int_{0}^{t_{1}} a_{q_{v}}\left(q_{v}^{\varepsilon}, q_{v}^{b \varepsilon}\right) d t \rightarrow \int_{0}^{t_{1}} a_{q_{v}}\left(q_{v}, q_{v}^{b}\right) d t .
$$


On the other hand, we can now pass to the lower limit in the remaining part of the $a_{q_{v}}$-term and we obtain

$$
\liminf _{\varepsilon \rightarrow 0} \int_{0}^{t_{1}} a_{q_{v}}\left(q_{v}^{\varepsilon}, q_{v}^{\varepsilon}\right) d t \geqslant \int_{0}^{t_{1}} a_{q_{v}}\left(q_{v}, q_{v}\right) d t .
$$

Then, using the expressions of the linear and trilinear forms $l$ and $b$, as in (4.10), (4.8), the convergence results in (i) and Lemma 4.6, we deduce, as $\varepsilon \rightarrow 0$ and for all $q_{v}^{b} \in \mathcal{K}$, that

$$
\begin{array}{r}
\int_{0}^{t_{1}} b\left(\mathbf{u}, q_{v}^{\varepsilon}, q_{v}^{b \varepsilon}-q_{v}^{\varepsilon}\right) d t \rightarrow \int_{0}^{t_{1}} b\left(\mathbf{u}, q_{v}, q_{v}^{b}-q_{v}\right) d t \\
\int_{0}^{t_{1}} l_{q_{v}}\left(q_{v}^{b \varepsilon}-q_{v}^{\varepsilon}\right) d t \rightarrow \int_{0}^{t_{1}} l_{q_{v}}\left(q_{v}^{b}-q_{v}\right) d t .
\end{array}
$$

In fact, the convergence result (4.114) is obvious. However, for (4.113), we used the following estimates:

$$
\begin{aligned}
& \left|\int_{0}^{t_{1}}\left[b\left(\mathbf{u}, q_{v}^{\varepsilon}, q_{v}^{b \varepsilon}-q_{v}^{\varepsilon}\right) d t-b\left(\mathbf{u}, q_{v}, q_{v}^{b}-q_{v}\right)\right] d t\right| \\
& \quad=\left(\left|\int_{0}^{t_{1}}\left[b\left(\mathbf{u}, q_{v}^{\varepsilon}-q_{v}, q_{v}^{b \varepsilon}-q_{v}^{\varepsilon}\right) d t+b\left(\mathbf{u}, q_{v}, q_{v}^{b \varepsilon}-q_{v}^{b}\right)-b\left(\mathbf{u}, q_{v}, q_{v}^{\varepsilon}-q_{v}\right)\right] d t\right|\right) \\
& \quad \leqslant|\mathbf{u}|_{L^{\infty}\left(\left(0, t_{1}\right) \times \mathcal{M}\right)}\left[\left|q_{v}^{\varepsilon}-q_{v}\right|_{L^{2}\left(0, t_{1} ; V\right)}+\left|q_{v}^{b \varepsilon}-q_{v}^{b}\right|_{L^{2}\left(0, t_{1} ; V\right)}\right] .
\end{aligned}
$$

Now for the RHS of (4.106), we use (4.101) together with (v) and Lemma 4.5 and we obtain

$$
\left(f_{q_{v}}\left(U^{\varepsilon}\right)+F \mathcal{H}_{\varepsilon_{2}}\left(q_{v}-q_{v s}\right), q_{v}^{b \varepsilon}-q_{v}^{\varepsilon}\right) \rightarrow\left(f_{q_{v}}(U)+F h_{q_{v}}, q_{v}^{b}-q_{v}^{\varepsilon}\right), \text { as } \varepsilon \rightarrow 0, \forall q_{v}^{b} \in \mathcal{K} .
$$

We are left to check that $h_{q_{v}}$ belongs to $\mathcal{H}\left(q_{v}-q_{v s}\right)$, i.e., to prove $(4.31)$. For $\varepsilon_{2} \in(0,1]$, we define the real function

$$
K_{\varepsilon_{2}}(r)= \begin{cases}0 & \text { for } r \leqslant 0, \\ r^{2} / 2 \varepsilon_{2} & \text { for } r \in\left(0, \varepsilon_{2}\right], \\ r-\varepsilon_{2} / 2 & \text { for } r>\varepsilon_{2} .\end{cases}
$$

It is straightforward to check that $K_{\varepsilon_{2}}^{\prime}=\mathcal{H}_{\varepsilon_{2}}$, and for $\forall r_{1}, r_{2} \in \mathbb{R}$

$$
\begin{aligned}
\left|\mathcal{H}_{\varepsilon_{2}}\left(r_{1}\right)-\mathcal{H}_{\varepsilon_{2}}\left(r_{2}\right)\right| & \leqslant \frac{1}{\varepsilon_{2}}\left|r_{1}-r_{2}\right|, \\
\left|K_{\varepsilon_{2}}\left(r_{1}\right)-K_{\varepsilon_{2}}\left(r_{2}\right)\right| & \leqslant\left|r_{1}-r_{2}\right| .
\end{aligned}
$$

Moreover,

$$
\left|K_{\varepsilon_{2}}(r)-r\right| \leqslant \frac{\varepsilon_{2}}{2}, \forall r \geqslant 0 .
$$

Becuase $\mathcal{H}_{\varepsilon_{2}}\left(q_{v}^{\varepsilon}-q_{v s}^{\varepsilon}\right)$ is the Gâteaux derivative at the point $q_{v}^{\varepsilon}-q_{v s}^{\varepsilon}$ of the convex function

$$
\int_{0}^{t_{1}}\left(K_{\varepsilon_{2}}(\cdot), 1\right) d t: L^{2}\left(0, t_{1} ; V\right) \rightarrow \mathbb{R}
$$


For every $\varepsilon$, we have

$$
\int_{0}^{t_{1}}\left(K_{\varepsilon_{2}}\left(q_{v}^{b \varepsilon}-q_{v s}^{\varepsilon}\right), 1\right) d t-\int_{0}^{t_{1}}\left(K_{\varepsilon_{2}}\left(q_{v}^{\varepsilon}-q_{v s}^{\varepsilon}\right), 1\right) d t \geqslant \int_{0}^{t_{1}}\left\langle\mathcal{H}_{\varepsilon_{2}}\left(q_{v}^{\varepsilon}-q_{v s}^{\varepsilon}\right), q_{v}^{b \varepsilon}-q_{v}^{\varepsilon}\right\rangle d t
$$

for each $q_{v}^{b} \in L^{2}\left(0, t_{1} ; V\right)$. By (v), (vii) and Lemma 4.6, we see that, as $\varepsilon \rightarrow 0^{+}$,

$$
\int_{0}^{t_{1}}\left\langle\mathcal{H}_{\varepsilon_{2}}\left(q_{v}^{\varepsilon}-q_{v s}^{\varepsilon}\right), q_{v}^{b \varepsilon}-q_{v}^{\varepsilon}\right\rangle d t \rightarrow \int_{0}^{t_{1}}\left\langle h_{q_{v}}, q_{v}^{b}-q_{v}\right\rangle d t
$$

for $\forall q_{v}^{b} \in L^{2}\left(0, t_{1} ; V\right)$.

Moreover, owing to (4.118) and (4.120), we observe that

$$
\begin{aligned}
& \left|\int_{0}^{t_{1}}\left(K_{\varepsilon_{2}}\left(q_{v}^{\varepsilon}-q_{v s}^{\varepsilon}\right), 1\right) d t-\int_{0}^{t_{1}}\left(\left[q_{v}-q_{v s}\right]^{+}, 1\right) d t\right| \\
& \leqslant \int_{0}^{t_{1}}\left(\mid\left(K_{\varepsilon_{2}}\left(q_{v}^{\varepsilon}-q_{v s}^{\varepsilon}\right)-K_{\varepsilon_{2}}\left(q_{v}-q_{v s}\right) \mid, 1\right) d t+\int_{0}^{t_{1}}\left(\left|K_{\varepsilon_{2}}\left(q_{v}-q_{v s}\right)-\left[q_{v}-q_{v s}\right]^{+}\right|, 1\right) d t\right. \\
& \leqslant \mu(\mathcal{M})^{1 / 2} t_{1}^{1 / 2}\left(\left|q_{v}^{\varepsilon}-q_{v}\right|_{L^{2}\left(0, t_{1} ; L^{2}\right)}+\left|q_{v s}^{\varepsilon}-q_{v s}\right|_{L^{2}\left(0, t_{1} ; L^{2}\right)}\right)+\frac{\varepsilon_{2}}{2} \mu(\mathcal{M}) t_{1}
\end{aligned}
$$

where $\mu(\mathcal{M})$ is the volume of $\mathcal{M}$. Therefore

$$
\left.K_{\varepsilon_{2}}\left(q_{v}^{\varepsilon}-q_{v s}^{\varepsilon}\right), 1\right) d t \rightarrow \int_{0}^{t_{1}}\left(\left[q_{v}-q_{v s}\right]^{+}, 1\right) d t
$$

From the calculation above, it is also clear that

$$
\left.K_{\varepsilon_{2}}\left(q_{v}^{b \varepsilon}-q_{v s}^{\varepsilon}\right), 1\right) d t \rightarrow \int_{0}^{t_{1}}\left(\left[q_{v}^{b}-q_{v s}\right]^{+}, 1\right) d t
$$

for $\forall q_{v}^{b} \in L^{2}\left(0, t_{1} ; V\right)$.

Consequently, we can pass to the limit in (4.121) and conclude that

$$
\int_{0}^{t_{1}}\left(\left[q_{v}^{b}-q_{v s}\right]^{+}, 1\right) d t-\int_{0}^{t_{1}}\left(\left[q_{v}-q_{v s}\right]^{+}, 1\right) d t \geqslant \int_{0}^{t_{1}}\left\langle h_{q_{v}}, q_{v}^{b}-q_{v}\right\rangle d t
$$

for $\forall q_{v}^{b} \in L^{2}\left(0, t_{1} ; V\right)$, which implies (4.31) as desired.

Finally, we obtain the existence of the solution of (4.28) for which we state the following theorem.

Theorem 4.8. Let $U_{0} \in \mathbb{V}, t_{1}>0$ be given and assume that $\mathbf{u} \in L^{\infty}\left(\left(0, t_{1}\right) \times \mathcal{M}\right)$ is given. Then, the system (4.25)-(4.28) associated with the initial and boundary conditions (4.34) and (4.35), respectively, has a solution $U$ such that

$$
U \in L^{\infty}\left(0, t_{1} ; \mathbb{H}\right) \cap L^{2}\left(0, t_{1} ; \mathbb{V}\right),
$$

Furthermore, we have

$$
\bar{U} \in L^{2}\left(0, t_{1} ; H^{2}\right), \quad \partial_{t} \bar{U} \in L^{2}\left(0, t_{1} ; L^{2}\right), \quad \partial_{t} q_{v} \in L^{5 / 3}\left(0, t_{1} ; V^{*}\right) .
$$


Acknowledgments. This work is partially supported by the National Science Foundation under the grants NSF-DMS-1510249 and by the Research Fund of Indiana University.

\section{REFERENCES}

[Bre72] Brézis, Hä̈m. Problèmes unilatéraux. J. Math. Pures Appl. (9) 51 (1972), 1-168. 2

[BF78] A. Bensoussan, A. Friedman, On the support of the solution of a system of quasi variational inequalities, J. Math. Anal. Appl. 65 (1978), 660-674. 10

[BL84] A. Bensoussan, J. L. Lions, Impulse control and quasivariational inequalities. Translated from the French by J. M. Cole. $\mu$. Gauthier-Villars, Montrouge; Heyden \& Son, Inc., Philadelphia, PA, 1984. $x i v+684$ pp. 10

[BL77] A. Bensoussan, J. L. Lions, Inéquations quasi variationnelles dépendant d'un paramètre, Ann. Scuola Norm. Sup. Pisa Cl. Sci. (4) 4 (1977), 231-255. 10

[BL74] A. Bensoussan, J. L. Lions, Contrôle impulsionnel et contrôle continu. Méthode des inéquations quasi variationnelles non linéaires. (French) C. R. Acad. Sci. Paris Sér. A 278 (1974), 675-679. 10

[BL73a] A. Bensoussan, J. L. Lions, Contrôle impulsionnel et inéquations quasi-variationnelles stationnaires. (French) C. R. Acad. Sci. Paris Sér. A-B 276 (1973), A1279-A1284. 10

[BL73b] A. Bensoussan, J. L. Lions, Nouvelle formulation de problèmes de contrôle impulsionnel et applications. (French) C. R. Acad. Sci. Paris Sér. A-B 276 (1973), A1189-A1192. 10

[BL75] A. Bensoussan, J. L. Lions, Nouvelles méthodes en contrôle impulsionnel. (French) Appl. Math. Optim. 1 (1974/75), no. 4, 289-312. 10

[BL76] A. Bensoussan, J. L. Lions, On the support of the solution of some variational inequalities of evolution, J. Math. Soc. Japan 28 (1976), 1-17. 10

[BCT14] A. Bousquet, M. Coti Zelati, R. Temam, Phase transition models in atmospheric dynamics, Milan J. Math. 82 (2014) 99-128. 1, 2, 3

[BCHTT15] A. Bousquet, M. Chekroun, Y. Hong, R. Temam and J. Tribbia, Numerical simulations of the humid atmosphere above a mountain, Math. Clim. Weather Forecast, 1, 2015, 96-126.

[Cea78] J. Cea, Lectures on optimization: theory and algorithms, Springer (1978) 6

[Cou43] R. Courant, Variational methods for the solution of problems of equilibrium and vibrations, Bull. Amer. Math. Soc, 49 (1943), 1-23. 6

[CT07] C. Cao, E.S. Titi. Global well-posedness of the three-dimensional viscous primitive equations of large scale ocean and atmosphere dynamics, Ann. of Math. (2) 166 (2007), 245-267. 4

[CHKTZ] M. Coti Zelati, A. Huang, I. Kukavica, R. Temam and M. Ziane, The primitive equations of the atmosphere in presence of vapor saturation, Nonlinearity, to appear. 2 2

[CFTT13] M. Coti Zelati, M. Fremond, R. Temam and J. Tribbia, The equations of the atmosphere with humidity and saturation: uniqueness and physical bounds, Physica D 264 (2013), 49-65. 1, 2, $6,10,14$

[CT12] M. Coti Zelati and R. Temam, The atmospheric equation of water vapor with saturation, Boll. Unione Mat. Ital., (9), 2012, 5, 309-336. 1, 2, 6, 10, 14

[Dia93] J. I. Díaz. Mathematical analysis of some diffusive energy balance models in Climatology. En el libro Mathematics, Climate and Environment (J. I. Díaz and J.L.Lions eds.), Research Notes in Applied Mathematics no 27, Masson, Paris, 2856, 1993. 1

[Dau88] M. Dauge, Elliptic boundary value problems on corner domains, Springer-Verlag, Berlin, 1988

[DT99] J. I. Díaz, L. Tello. On a nonlinear parabolic problem on a Riemannian manifold without boundary arising in Climatology. Collectanea Mathematica, Volum L, Fascicle 1, 19-51, 1999. 1

[DL76] G. Duvaut, J.-L. Lions, Inequalities in Mechanics and Physics, translated from the French by C.W. John Springer-Verlag, BerlinNew York, 1976, xvi+397 pp. 2

[ET76] I. Ekeland and R. Temam, Convex analysis and variational problems. Translated from the French. Studies in Mathematics and its Applications, Vol. 1. North-Holland Publishing Co., Amsterdam-Oxford; American Elsevier Publishing Co., Inc., New York, 1976. ix+402 pp. 2 
[Fei91] E. Feireisl, A note on uniqueness for parabolic problems with discontinuous nonlinearities, Nonlinear Anal. 16 (1991), 1053-1056. 1

[Fre02] Michel Frémond, Non-Smooth Thermomechanics (English Summary), Springer-Verlag, Berlin, 2002, xvi+480 pp. 2

[FN91] E. Feireisl, J. Norbury, Some existence, uniqueness and nonuniqueness theorems for solutions of parabolic equations with discontinuous nonlinearities, Proc. Roy. Soc. Edinburgh Sect. A 119 (1991), 1-17. 1

[Gil82] A. E. Gill, Atmosphere-Ocean Dynamics, International Geophysics Series, Volume 30, Academic Press, 1st Edition, 1982, 662 pages. 1

[Gra98] W. W. Grabowski, Toward cloud resolving modeling of large-scale tropical circulations: a simple cloud microphysics parametrization, Journal of the Atmospheric Sciences, 55 (1998), 32833298. 2

[GH06] B. Guo and D. Huang, Existence of weak solutions and trajectory attractors for the moist atmospheric equations in geophysics, J. Math. Phys. 47 (2006), 083508, 23.1

[GH11] B. Guo and D. Huang, Existence of the universal attractor for the 3-D viscous primitive equations of large-scale moist atmosphere, J. Differential Equations 251 (2011), 457-491. 1

[Hal71] G. J. Haltiner, Numerical Weather Prediction, John Wiley and Sons, New York, 1971. 2, 4

[HKLT17] S. Hittmeir, R. Klein, J. Li, and E. S. Titi, Global well-posedness for passively transported nonlinear moisture dynamics with phase changes, Nonlinearity 30 (2017), no. 10, 3676-3718. 2, 6

[HW80] G. J. Haltiner and R. T. Williams, Numerical prediction and dynamic meteorology, John Wiley and Sons, New York, 1980. 2, 4

[Kan14] R. Kano, The existence of solutions for tumor invasion models with time and space dependent diffusion. Discrete Contin. Dyn. Syst. Ser. S 7 (2014), no. 1, 63-74. 10

[Kes69] E. Kessler, On the distribution and continuity of water substance in atmospheric circulation, Meteorological Monographs, American Meteorological Society, 1969.

[Kob06] G. M. Kobelkov, Existence of a solution 'in the large' for the 3D large-scale ocean dynamics equations, C. R. Math. Acad. Sci. Paris, 343 (2006), 283-286. 2

[Kob07] G. M. Kobelkov, Existence of a solution 'in the large' for ocean dynamics equations, J. Math. Fluid Mech, 9 (2007), 588-610.

[KM06] R. Klein and A. J. Majda, Systematic multiscale models for deep convection on mesoscales, Theor. Comput. Fluid Dyn. 20 (2006), 525-551. 2

[KN07] A. S. Kravchuk, P. J. Neittaanmki $\ddot{a}$, Variational and quasi-variational inequalities in mechanics, Solid mechanics and its application, Vol. 147, 2007, 345 pages. 10

[KS80] D. Kinderlehrer and G. Stampacchia, An introduction to variational inequalities and their applications, Academic Press Inc. [Harcourt Brace Jovanovich Publishers], New York, 1980.

[KS80] D. Kinderlehrer, G. Stampacchia, An Introduction to Variational Inequalities and Their Applications, Academic Press Inc., Harcourt Brace Jovanovich Publishers, New York, 1980. 2

[KW78] J. B. Klemp, R. B. Wilhelmson, The Simulation of Three-Dimensional Convective Storm Dynamics, J. Atmos. Sci. 35 (1978), 1070-1096. 2, 3, 4, 5

[KBH98] T. N. Krishnamurti, H. S. Bedi, V. M. Hardiker, An Introduction to Global Spectral Modeling, Oxford University Press, 1st edition, 1998. 2

[Lio76] J.L. Lions, Quelques Méthodes de Résolution des Problèmes aux Limites Non Linéaires, Dunod, Paris, 1969. 28

[LLBS14] F. Lenzen, J. Lellmann, F. Becker, and C. Schnrr, Solving quasi-variational inequalities for image restoration with adaptive constraint sets. SIAM J. Imaging Sci. 7 (2014), no. 4, 2139-2174. 10

[LM72] J.-L. Lions and E. Magenes, Non-homogeneous boundary value problems and applications. Vol. I, Springer-Verlag, New York, 1972

[LTW92] J. L. Lions, R. Temam and S. Wang, New formulations of the primitive equations of atmosphere and applications. Nonlinearity 5 (1992), no. 2, 237-288. 1

[Mil14] M. Milasi, Existence theorem for a class of generalized quasi-variational inequalities. J. Global Optim. 60 (2014), no. 4, 679-688. 10

[Mos78] J. Mossino. Application des inéquations quasi-variationnelles à quelques problèmes non linéaires de la physique des plasmas. (French. English summary) Israel J. Math. 30 (1978), no. $1-2,14-50$. 
[Mos77] J. Mossino. Étude d'une inéquation quasi-variationnelle apparaissant en physique, Convex analysis and its applications (Proc. Conf., Muret-le-Quaire, 1976). 139-157. Lecture Notes in Econom. and Math. Systems, Vol. 144. Springer, Berlin, 1977.

[Mos76] J. Mossino. Sur certaines inéquations quasi-variationnelles apparaissant en physique. $C . R$. Acad. Sci. Paris Sér. A-B, 282, 1976.

[MM76] M. W. Moncrieff, M. J. Miller, The dynamics and simulation of tropical cumuloniumbus and squall lines, Quarterly Journal Royal Meteorological Society, 102 (1976), 373-394.

[MP74] M. J. Miller, R. P. Pearce, A three-dimensional primitive equation model of cumulonimbus convection, Quarterly Journal Royal Meteorological Society, 100 (1974), 133-154. 3, 5

[MW84], M. J. Miller, A. A. White, On the non-hydrostatic equations in pressure and sigma coordinates, Quar. J. R. Met. Soc., 110 (1984), 515-533.

[Ped87] J. Pedlosky, Geophysical fluid dynamics, Springer-Verlag, New York, 1987. 1

[Pet06] M. Petcu, On the three-dimensional primitive equations, Adv. Differential Equations 11 (2006), 1201-1226.

[Pry81] A. J. Pryde, Second order elliptic equations with mixed boundary conditions, J. Math. Anal. Appl. 80 (1981), 203-244.

[PT80] E. Polak, A. L. Tits, A globally convergent, implementable multiplier method with automatic penalty limitation, Appl Math Optim. 6 (1980), 335-360. 6

[RY89] R. R. Rogers, M. K. Yau, A Short Course in Cloud Physics, Pergamon Press, Oxford, New York, 1989. 2, 4

[SO73] S. Soong, Y. Ogura, A comparison between axisymmetric and slab-symmetric cumulus cloud models, Journal of the Atmospheric Sciences, 30 (1973), 879-893.

[Tem95] Roger Temam. Navier-Stokes equations and nonlinear functional analysis. SIAM, Philadelphia, 1995.

[TT16] R. Temam and J. Tribbia, The equations of moist advection: a unilateral problem. Quarterly Journal of the Royal Meteorological Society 142 (2016), 143-146. 2, 13

[TWa15] R. Temam, X. Wang, Approximation of the equations of the humid atmosphere with saturation, to appear. 1, 2, 3, 20, 28

[TWu15] R. Temam, K. Wu, Formulation of the equations of the humid atmosphere in the context of variational inequalities, Journal of Functional Analysis, 269 (2015), 2187-2221. 1, 2, 3, 14, 20, 28

[Xue89] M. Xue, A nonhydrostatic numerical model in coordinates and simulations of mesoscale phenomena, Ph.D. Thesis, Department of Meteorology, University of Reading, 1989, 258 pages. 2,5

[Kar11] V.G. Karmanov (originator), Method of penalty function, in Encyclopedia of Mathematics. URL: https://www.encyclopediaofmath.org/index.php/Penalty_functions,_method_of, 2011 (accessed November 6, 2017). 6

$\dagger$ Indiana University, Institute for Scientific Computing and Applied Mathematics, Rawles Hall, Bloomington, in 47405, USA., ‡ National Center for Atmospheric Research, Boulder, Colorado. * University of Tunis El Manar, Facutly of Sciences of Tunis, Department of Mathematics, Tunis, Tunisia.

E-mail address: ${ }^{\dagger}$ yinicao@indiana.edu (Y. Cao), ${ }^{\dagger}$ mahamoud@indiana.edu (M. Hamouda),

$\dagger$ temam@indiana.edu (R. Temam), ${ }^{\dagger}$ tribbia@ucar.edu (J. Tribbia),

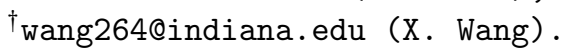

